\title{
Ultra-deformable lipid vesicles localise Amphotericin B in the dermis for the treatment of skin infectious diseases
}

Raquel Fernández-García ${ }^{1 \#, ~ L a r r y ~ S t a t t s ~}{ }^{2 \#}$, Jéssica A. de Jesus ${ }^{3}$, Maria Auxiliadora DeaAyuela $^{4}$, Liliana Bautista ${ }^{2}$, Rúben Simão ${ }^{1}$, Francisco Bolás-Fernández ${ }^{5}$, Maria Paloma Ballesteros $^{1,6}$, Marcia Dalastra Laurenti ${ }^{3}$, Luiz F. D. Passero ${ }^{7,8}$, Aikaterini Lalatsa ${ }^{2 *}$, Dolores R. Serrano ${ }^{1,6 *}$.

${ }^{1}$ Departamento de Farmacia Galénica y Tecnología Alimentaria, Facultad de Farmacia, Universidad Complutense de Madrid. Plaza Ramón y Cajal s/n, 28040, Madrid (Spain)

${ }^{2}$ Biomaterials, Bio-engineering and Nanomedicines (BioN) laboratory, Institute of Biomedical and Biomolecular Sciences, School of Pharmacy and Pharmaceutical Sciences, University of Portsmouth, St. Michael's Building, White Swan Road, Portsmouth (United Kingdom)

${ }^{3}$ Laboratory of Pathology of Infectious Diseases (LIM-50), Medical School, University of São Paulo, Avenida Dr. Arnaldo 455, 01246903 Cerqueira César, SP (Brazil)

${ }^{4}$ Departamento de Farmacia. Facultad de Ciencias de la Salud. Universidad CEU Cardenal Herrera. Carrer Santiago Ramón y Cajal, s/n. 46113 Valencia (Spain)

${ }^{5}$ Departamento de Microbiología y Parasitología, Facultad de Farmacia, Universidad Complutense de Madrid. Plaza Ramón y Cajal s/n, 28040, Madrid (Spain)

${ }^{6}$ Instituto Universitario de Farmacia Industrial, Facultad de Farmacia, Universidad Complutense de Madrid. Plaza Ramón y Cajal s/n, 28040, Madrid (Spain)

${ }^{7}$ São Paulo State University (UNESP), Institute of Biosciences, São Vicente. Praça Infante Dom Henrique, s/n, 11330-900 São Vicente, SP, (Brazil)

${ }^{8}$ São Paulo State University (UNESP), Institute for Advanced Studies of Ocean, São Vicente. Av. João Francisco Bensdorp, 1178, 11350-011 São Vicente, SP (Brazil)

\# First co-authorship shared

*Corresponding authors:

Dr Aikaterini Lalatsa

School of Pharmacy and Biomedical Sciences

University of Portsmouth

White Swan Road,

Portsmouth, PO1 2DT

Email: katerina.lalatsa@port.ac.uk

Tel: +442392843929

Dolores R. Serrano

Departamento de Farmacia Galénica y Tecnología Alimentaria

Facultad de Farmacia

Universidad Complutense de Madrid

28040-Madrid, Spain

Email: drserran@ucm.es

Tel: +34913941620 


\begin{abstract}
Cutaneous fungal and parasitic diseases remain challenging to treat, as available therapies are unable to permeate the skin barrier. Thus, treatment options rely on systemic therapy, which fail to produce high drug local concentrations but can lead to significant systemic toxicity. Amphotericin B (AmB) is highly efficacious in the treatment of both fungal and parasitic diseases such as cutaneous leishmaniasis, but is only reserved for parenteral administration in patients with severe pathophysiology. Here, we have designed and optimised AmB-transfersomes [93.5\% encapsulation efficiency, size of $150 \mathrm{~nm}$, and good colloidal stability $(-35.02 \mathrm{mV})]$ that can remain physicochemically stable $(>90 \%$ drug content) at room temperature and $4{ }^{\circ} \mathrm{C}$ over 6 months when lyophilised and stored under desiccated conditions. AmBtransfersomes possessed good permeability across mouse skin $\left(4.91 \pm 0.41 \mu \mathrm{g} / \mathrm{cm}^{2} / \mathrm{h}\right)$ and 10 -fold higher permeability across synthetic Strat- $\mathrm{M}^{\circledR}$ membranes. In vivo studies after a single topical application in mice showed permeability and accumulation within the dermis $(>25 \mu \mathrm{g} \mathrm{AmB} / \mathrm{g}$ skin at $6 \mathrm{~h}$ post-administration) indicating the delivery of therapeutic amounts of AmB for mycoses and cutaneous leishmaniasis, while a single daily administration in Leishmania (Leishmania) amazonensis infected mice over 10 days resulted in excellent efficacy (98\% reduction in Leishmania parasites). Combining the application of AmB-transfersomes with metallic microneedles in vivo increased levels in the SC and dermis but is unlikely to elicit transdermal levels. In conclusion, AmB-transfersomes are promising and stable topical nanomedicines that can be readily translated for parasitic and fungal infectious diseases.
\end{abstract}

\title{
KEYWORDS
}

Amphotericin B, transfersomes, ultra-deformable lipid vesicles, fungal infections, leishmaniasis, tape stripping 
Skin infections are a common public health problem in developed and developing countries. However, they remain challenging to treat as available therapies are unable to permeate the skin barrier and localise the drug within the skin at high concentration without the need of frequent administrations or in some severe infections such as cutaneous and mucocutaneous leishmaniasis of prolonged intralesional administration. Thus, these patients rely on systemic therapy (oral or intravenous) that fails to result in high local concentration in the skin, while able to lead to significant systemic toxicity.

Amphotericin B (AmB) is a macrolide chemotherapeutic produced by Streptomyces nodosus ${ }^{1}$ that has been the "gold-standard" of the antifungal therapy since its commercialisation, while remaining the first line therapy in the developed world for the treatment of leishmaniasis ${ }^{2}$. Although highly effective with limited occurrence of resistance, $\mathrm{AmB}$ is a large, amphiphilic, poorly water-soluble drug (below 0.001 $\mathrm{mg} / \mathrm{ml}$ ) with poor permeability across biological barriers ${ }^{1,3}$. AmB is currently only licensed as a liposomal formulation for parenteral administration $\left(\mathrm{AmBisome}^{\odot}\right.$ ) as older micellar formulations are highly nephrotoxic. AmBisome ${ }^{\odot}$ apart from being highly expensive and thus not really an option for the treatment of neglected parasitic diseases such as cutaneous and mucocutaneous leishmaniasis is also highly unstable at room temperature ${ }^{4}$. A cream containing $3 \%$ of AmB (Anfoleish) for topical use is currently under clinical trials (NCT01845727), but preliminary results have shown variable efficacy in patients with cutaneous leishmaniasis which can be the result of a limited permeability across the skin 5. In addition, a range of adverse effects such as itching, redness, peeling dryness and irritation of the skin were manifested in treated patients with Anfoleish ${ }^{6}$.

Ultra-deformable lipid vesicles, also known as transferosomes or transfersomes (TFs), are drug delivery systems with shown potential to overcome the stratum corneum and reach deeper regions of the skin promoting drug targeting, while minimizing adverse effects ${ }^{7-9}$. TFs consist of lipids, an edge activator and a small amount of ethanol $(<10 \%)^{8}$ forming ultradeformable vesicles that permeate the skin following the natural water osmotic gradient ${ }^{10}$. Previous reports to develop AmB-TFs demonstrated their capability in vitro but in vivo proof of concept has not been shown ${ }^{11}$. The hypothesis underpinning this work is that the encapsulation of AmB within TFs can enable the AmB permeation across the skin and localise it in the epidermis and dermis in effective concentrations for the treatment of fungal and parasitic infections in a stable formulation for topical application. In this respect, we optimised AmBTFs using quality by design (QbD) to ensure high entrapment efficiency, small size and colloidal stability and assess their stability at room temperature and under refrigeration after prepared formulations were lyophilised and stored under desiccated conditions. To understand their permeation across the skin and drug localisation, we undertook ex vivo Franz cell diffusion assays across mouse skin and in vivo tape stripping experiments in mice. The efficacy of the formulation was demonstrated in Candida albicans, C glabrata and C. parapsilosis disk diffusion assays and in vitro efficacy against Leishmania (Leishmania) amazonensis and Leishmania (Viannia) braziliensis promastigotes and amastigotes, while their in vivo activity was also demonstrated in $L$. (L.) amazonensis infected mice 
treated with AmB-TFs. Finally, in vitro toxicity in murine macrophages, haemolysis and histopathology studies were performed to demonstrate the safety of the proposed transcutaneous formulation.

\section{RESULTS}

\section{Design of experiments (DOE) studies and optimisation of AmB-TFs}

The 17 experimental runs including independent and dependent variables are shown in table 1.

Table 1. Independent variables and responses of all tested formulations.

\begin{tabular}{|c|c|c|c|c|c|c|}
\hline \multirow{2}{*}{ Run } & \multicolumn{3}{|c|}{ Independent variables } & \multicolumn{3}{c|}{ Dependent variables } \\
\cline { 2 - 7 } & $\begin{array}{c}\text { Amount of } \\
\text { surfactant } \\
(\mathbf{\% )}\end{array}$ & $\begin{array}{c}\text { Amount of } \\
\text { drug (mg) }\end{array}$ & $\begin{array}{c}\text { Amount of } \\
\text { ethanol } \\
\mathbf{( \% )}\end{array}$ & $\begin{array}{c}\text { Particle } \\
\text { size (nm) }\end{array}$ & $\begin{array}{c}\text { Zeta } \\
\text { potential } \\
(\mathbf{m V})\end{array}$ & $\begin{array}{c}\text { Entrapment } \\
\text { efficiency } \\
(\%)\end{array}$ \\
\hline 1 & 6 & 1.25 & 8.5 & 107.8 & -25.11 & 66.62 \\
\hline 2 & 6 & 1.25 & 0 & 190.3 & -27.96 & 82.08 \\
\hline 3 & 13 & 2 & 0 & 806 & -36.74 & 67.46 \\
\hline 4 & 13 & 0.5 & 8.5 & 823 & -31.98 & 77.52 \\
\hline 5 & 13 & 1.25 & 4 & - & -49.76 & 91.1 \\
\hline 6 & 13 & 1.25 & 4 & 145.7 & -29.95 & 80.1 \\
\hline 7 & 6 & 0.5 & 4 & 118.2 & -27.67 & 69.59 \\
\hline 8 & 20 & 2 & 4 & 363 & -20.68 & 42.55 \\
\hline 9 & 13 & 1.25 & 4 & 88.2 & -34 & 82.16 \\
\hline 10 & 13 & 0.5 & 0 & 204 & -34.03 & 94.63 \\
\hline 11 & 20 & 1.25 & 8.5 & 351 & -33.78 & 77.4 \\
\hline 12 & 13 & 2 & 8.5 & 556 & -30.93 & 56.87 \\
\hline 13 & 20 & 0.5 & 4 & 1314 & -33.63 & 32.68 \\
\hline 14 & 20 & 1.25 & 0 & 92.2 & -35.82 & 56.5 \\
\hline 15 & 13 & 1.25 & 4 & 99.2 & -31.25 & 95.06 \\
\hline 16 & 13 & 1.25 & 4 & 138.1 & -33.63 & 78.45 \\
\hline 17 & 6 & 2 & 4 & 1091 & -20.16 & 44.9 \\
\hline
\end{tabular}

Polynomial analysis was carried out by a multilinear regression analysis method suggesting that the quadratic model was the best fit for particle size and entrapment efficiency (EE). The coefficients of the model equations generated for each critical quality attribute (Eq. 1 \& 2 in S1.1 in SI) revealed goodness of fit (Fig. S1 in SI) of the experimental data to the selected model with high values of $\mathrm{R}^{2}(0.9772$ and 0.898 respectively) and low p-values $<0.05$ (Table S1 in SI). None of the three factors evaluated showed a significant impact on the zeta potential and possessed a zeta potential lower than $-20 \mathrm{mV}$ (Table 1). Thus, zeta potential was not utilised to inform the optimisation of the AmB-TFs. The multilinear regression models for particle size and EE are described in supplementary material.

The amount of AmB and sodium deoxycholate have a positive effect on particle size (Fig. 1a-c, Fig. S2-S4 in SI). The greater the amount, the larger the particle size of the TFs. The lowest particle size was achieved with low-intermediate amounts of sodium deoxycholate (6-12\%) and a drug content below $1.8 \mathrm{mg}$ per batch (Fig. S2 and S3 in SI). 
In terms of EE, intermediate amounts of edge activator and $\mathrm{AmB}$ resulted in the greatest $\mathrm{EE}$. The effect of the ethanol was not as sharply defined as a larger EE was obtained when using either a low or a high percentage $(<2 \%$ or $>7 \%)$ (Fig. 1d-f, Fig. S5-S7 in SI).
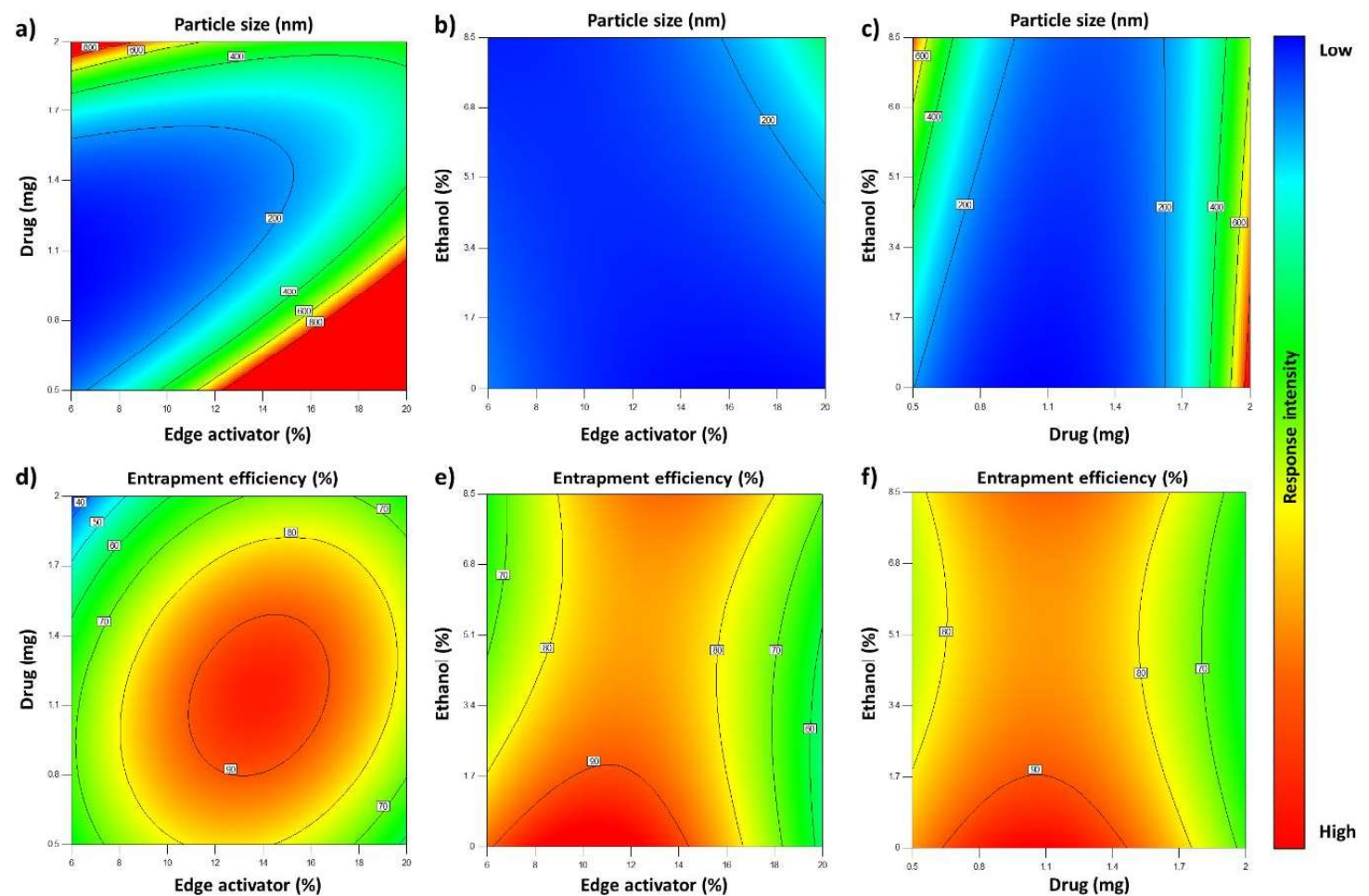

Figure 1. 2D Contour plots showing the effect of the amount of edge activator, drug and ethanol on particle size $(a, b, c)$ and EE (d, e, f).

The predicted and experimental values were in good agreement for particle size and EE. The optimised formulation consisted of 14.13:86.87 (w:w) sodium deoxycholate : soya phosphatidylcholine. The final drug loading after removing the unencapsulated fraction was $0.086 \%$. The reconstitution buffer was consisted of $91.5 \%$ PBS buffer (pH 7.4) and $8.5 \%$ of ethanol. Even though, a greater EE was obtained with amounts of ethanol below $2 \%$ or above $7 \%$, a greater amount of ethanol was chosen to enhance skin permeation. The optimised AmB-TFs resulted in a median particle size of $149 \pm 22 \mathrm{~nm}$ (D10 of $130 \pm 12 \mathrm{~nm}$ and a D90 of $168 \pm 6 \mathrm{~nm}$ ), with a zeta-potential of $-35.02 \pm 2.71 \mathrm{mV}$ and an EE of $93.3 \pm$ $1.9 \%$. The prediction error was well-below $10 \%$. No statistical difference was observed between fresh and lyophilised AmB-TFs in terms of particle size and zeta-potential ( $p>0.05$, Student t-test).

\section{Characterisation of optimised AmB-TFs}

TEM images of freshly prepared AmB-TFs (Fig. 2a) showed quasispherical particles characterised by a dark core and a lighter layer indicative of the phospholipid bilayer. Reconstitution of freeze dried AmB-TFs resulted in quasi-spherical particles with darker cores (Fig. 2b). However, the phospholipid 
bilayer was not as clearly observed as in the freshly prepared AmB-TFs. Similar morphology was observed after 28 days of storage at $4{ }^{\circ} \mathrm{C}$ (Fig. 2c).

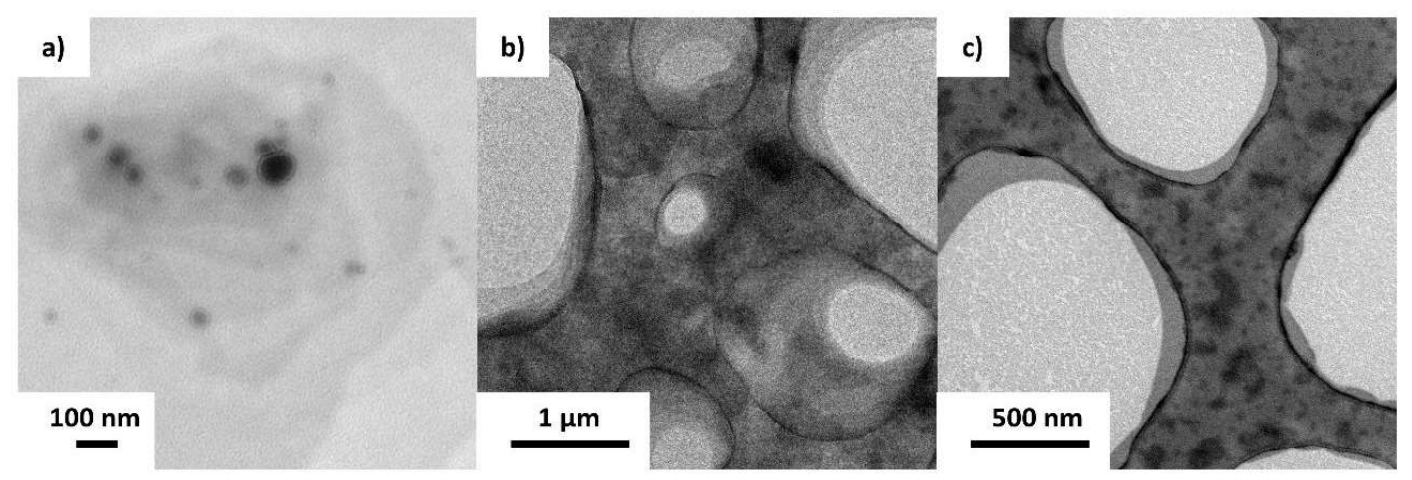

Figure 2. TEM micrographs of AmB-TFs; a) Freshly prepared AmB-TFs stained with $1 \%$ uranyl acetate (Bar: $100 \mathrm{~nm}$ ); b) Reconstituted freshly prepared freeze-dried AmB-TFs in deionised water stained with $2 \%$ phosphotungstic acid (Bar: $1 \mu \mathrm{m}$ ); c) AmB-TFs after 28 days of storage at $4{ }^{\circ} \mathrm{C}$ stained with $2 \%$ phosphotungstic acid (Bar: $500 \mathrm{~nm}$ ).

\section{Physicochemical stability of AmB-TFs}

Stability studies showed that AmB-TFs were physicochemically stable for at least one month in liquid state at $4{ }^{\circ} \mathrm{C}$ and for at least six months after freeze-drying at $4{ }^{\circ} \mathrm{C}$ and $25{ }^{\circ} \mathrm{C}$ under desiccated conditions $(10 \% \mathrm{RH})$, with a drug content higher than $90 \%$ (Fig. 3a \& 3c). The chemical degradation of AmBTFs was significantly affected when stored at $60 \%$ RH. Particle size of AmB-TFs was maintained over one month without significant differences between 4 and $25{ }^{\circ} \mathrm{C}$ storage temperature (Fig. 3b); however, reconstituted freeze dried AmB-TFs showed a tendency for aggregation at all the three tested conditions $\left(4^{\circ} \mathrm{C}, 25^{\circ} \mathrm{C} / 10 \% \mathrm{RH}\right.$ and $\left.25^{\circ} \mathrm{C} / 60 \% \mathrm{RH}\right)$ (Fig. $3 \mathrm{~d}$ ), being more pronounced at $25^{\circ} \mathrm{C}$ and $60 \% \mathrm{RH}$ (with 2-fold increase in size after 6 months, $\mathrm{p}<0.05$ ). This indicates that desiccated conditions are necessary to prevent physical instability of freeze dried AmB-TFs. 


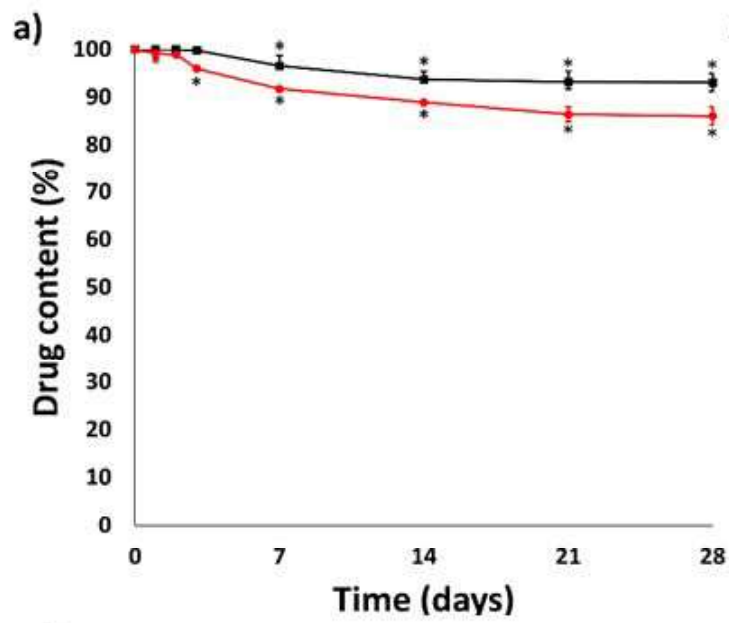

c)

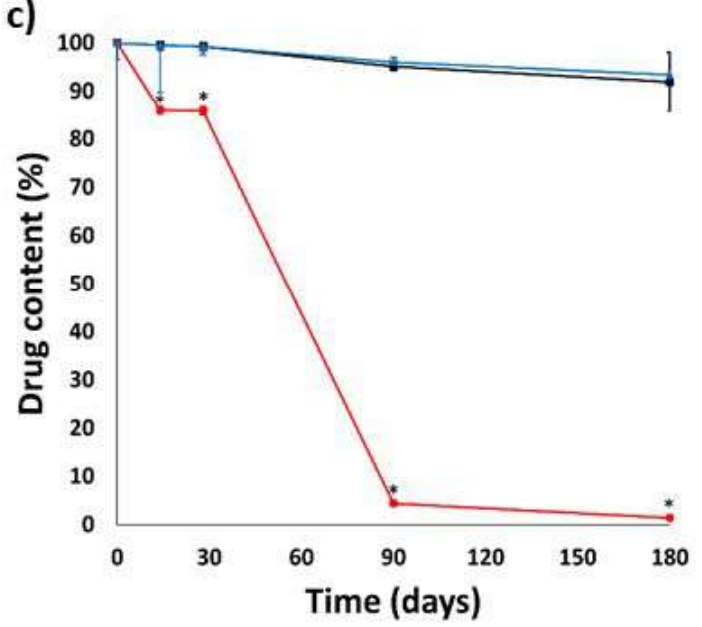

b)

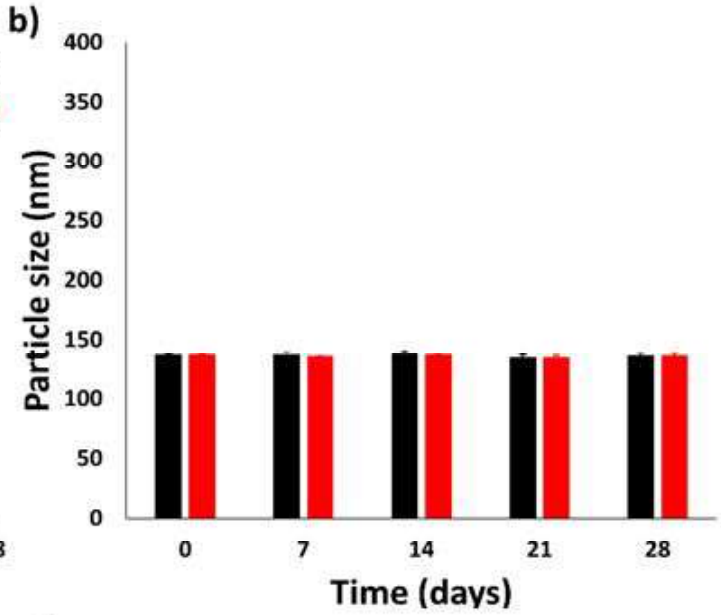

d)

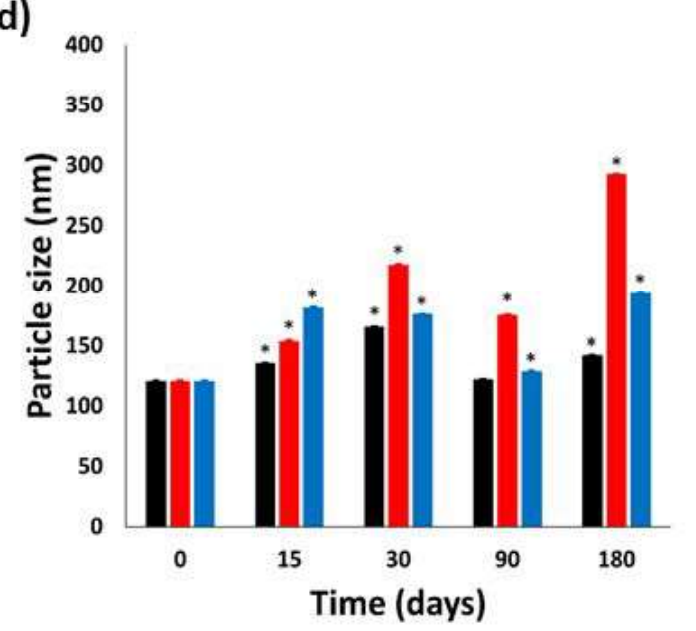

Figure 3. Physicochemical stability of liquid and freeze-dried AmB-TFs: a) Chemical stability of liquid AmB-TFs; b) Physical stability of liquid AmB-TFs. Key: $25{ }^{\circ} \mathrm{C}$ (red circles), $4{ }^{\circ} \mathrm{C}$ (black squares); ${ }^{*} \mathrm{p}<0.05$ is indicative of statistically significant difference between each time point and time 0 (one-way ANOVA post-hoc Dunnett's test). c) Chemical stability of freeze-dried AmB-TFs; d) Physical stability of freeze-dried AmB-TFs. Key: $25{ }^{\circ} \mathrm{C}, 10 \% \mathrm{RH}$ (black squares), $25{ }^{\circ} \mathrm{C}, 60 \% \mathrm{RH}$ (red circles), $4{ }^{\circ} \mathrm{C}, 10 \% \mathrm{RH}$ (blue triangles); ${ }^{*} \mathrm{p}<0.05$ is indicative of statistically significant difference between each time point and time 0 (one-way ANOVA post-hoc Dunnett's test).

\section{In vitro permeability studies}

AmB permeation across Strat-M ${ }^{\circledR}$ membranes was linear for both AmB-TFs and AmB-DMSO. Lag time observed was $9.99 \pm 4.59$ and $4.86 \pm 2.87$ minutes for AmB-TFs and AmB-DMSO, respectively. The steady flux over 6 hours for AmB-TFs was $41.18 \pm 1.39 \mu \mathrm{g} / \mathrm{cm}^{2} / \mathrm{h}$ and $211.06 \pm 15.33 \mu \mathrm{g} / \mathrm{cm}^{2} / \mathrm{h}$ for AmB-DMSO. It was found that AmB-DMSO had 5.13 times higher permeation than AmB-TFs $(\mathrm{p}=0.0018)$ across synthetic membranes (Fig. 4a). On the contrary, the two formulations started to permeate immediately across mouse skin, with no lag time. Flux was linear between times 0 to 250 
minutes and was $4.91 \pm 0.41 \mu \mathrm{g} / \mathrm{cm}^{2} / \mathrm{h}$ for AmB-TFs and $5.49 \pm 1.18 \mu \mathrm{g} / \mathrm{cm}^{2} / \mathrm{h}$ for AmB-DMSO. There were no statistically significant differences at permeation any point ( $p>0.05)$ (Fig. $4 b)$. Diffusion for AmB-DMSO was 17-fold lower across mouse skin than in Strat-M ${ }^{\circledR}$ membranes, while for AmB-TFs it was only 1.4-fold lower (Table 2). The amount of AmB entrapped in mouse skin after permeation studies was $3.28 \pm 0.84$ and $25.57 \pm 4.42 \mu \mathrm{g} \mathrm{AmB} / \mathrm{cm}^{2}$ skin for AmB-TFs and AmB-DMSO respectively. This illustrates the potential of TFs to deliver large molecular weight drugs such as AmB across the skin.

a)

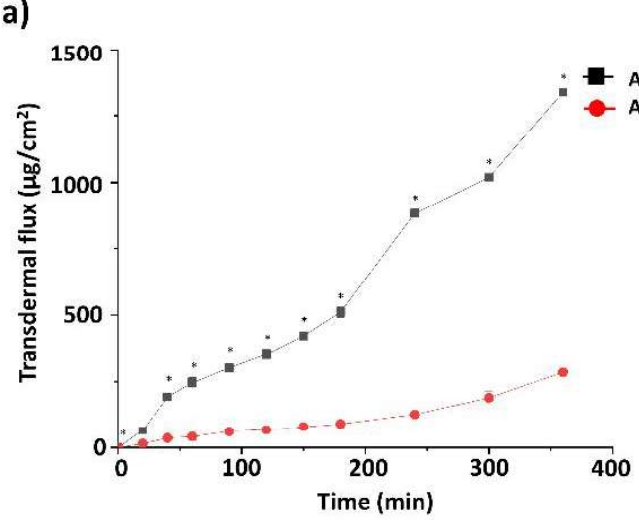

c)

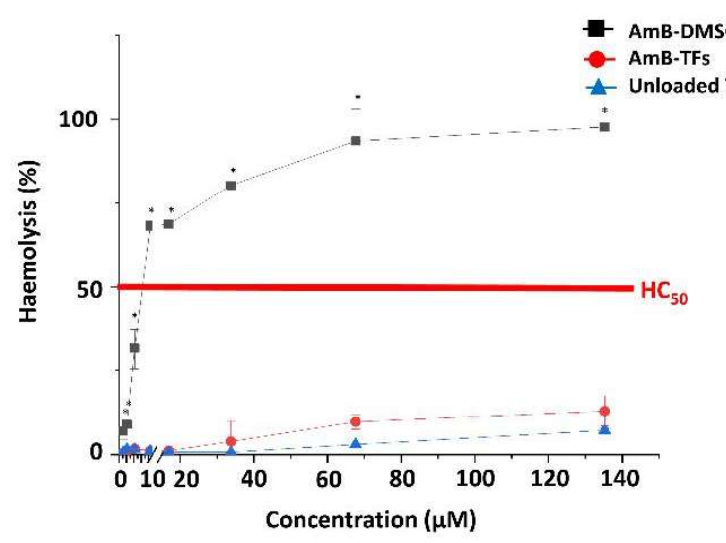

b)

d)
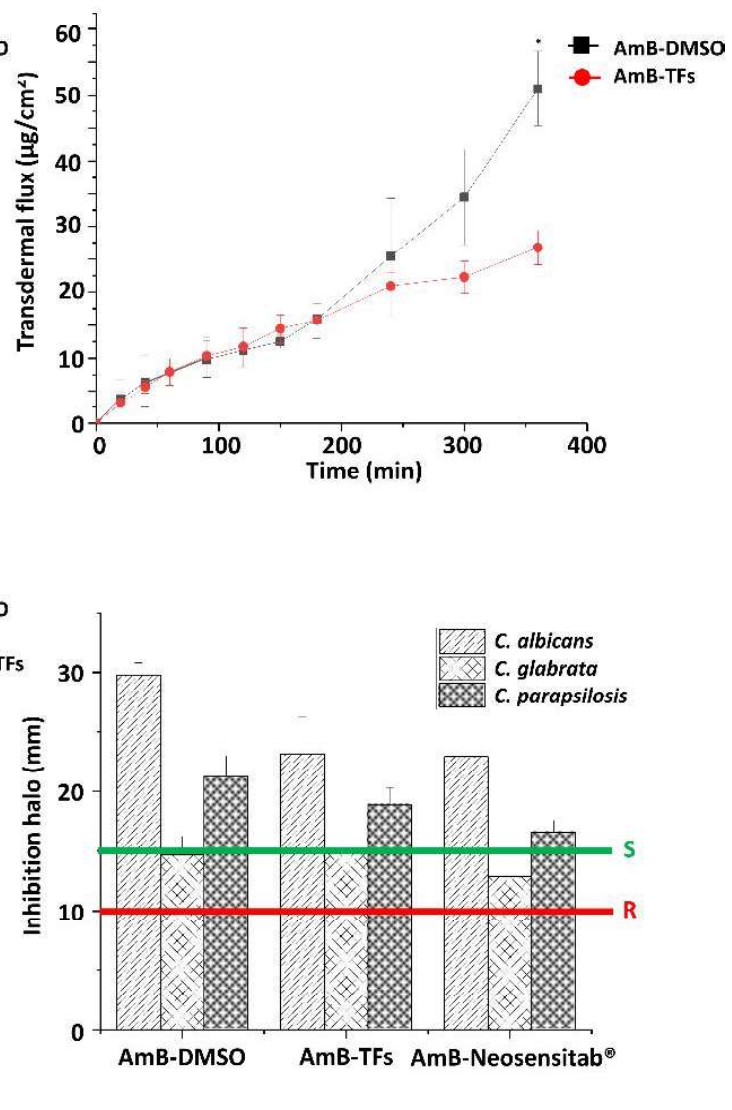

Figure 4. Permeability, toxicity and in vitro activity of AmB-TFs: a) Permeability of AmB-TFs and AmB-DMSO across Strat-M ${ }^{\circledR}$ membranes; b) Permeability of AmB-TFs and AmB-DMSO across mouse skin; c) Haemolytic toxicity of AmB-TFs, AmB-DMSO and unloaded TFs; d) Antifungal activity of AmB-TFs, AmB-DMSO and AmB-Neosensitabs ${ }^{\circledR}$. Key: AmB-DMSO (black squares), AmB-TFs (red circles), unloaded TFs (blue triangles), concentration that produces $50 \%$ of haemolysis $\left(\mathrm{HC}_{50}\right)$, sensitivity $(\mathrm{S})$, resistance $(\mathrm{R})$. Statistically significant difference is represented by $*(\mathrm{p}<0.05)$ when comparing to AmB-DMSO with other formulations.

Table 2. Permeability and diffusion across Strat- $\mathrm{M}^{\circledR}$ membranes and mouse skin for AmB-TFs and AmB-DMSO. 


\begin{tabular}{|c|c|c|c|}
\hline Formulation & Membrane & Permeability $(\mathrm{cm} / \mathrm{h})$ & Diffusion $\times 10^{-3}\left(\mathrm{~cm}^{2} / \mathrm{h}\right)$ \\
\hline \multirow{2}{*}{ AmB-TFs } & Strat-M ${ }^{\circledR}$ membrane & 0.075 & 2.25 \\
\hline & Mouse skin & $8.99 \times 10^{-3}$ & 1.6 \\
\hline \multirow{2}{*}{ AmB-DMSO } & Strat-M ${ }^{\circledR}$ membrane & 0.408 & 12.0 \\
\hline & Mouse skin & 0.011 & 0.711 \\
\hline
\end{tabular}

\section{Haemolysis assay}

Haemolytic studies for $\mathrm{AmB}$ in DMSO showed an $\mathrm{HC}_{50}$ of $10.93 \mu \mathrm{M}$, while values 10-fold higher (> $135 \mu \mathrm{M})$ were observed for both AmB-TFs and unloaded TFs. This suggests that entrapment of AmB in TFs is able to reduce the haematoxicity of AmB. (Fig. 4c).

\section{In vitro antifungal activity}

AmB-TFs were effective against the three strains of Candida spp. assayed (Fig. 4d). Similar to AmB dissolved in DMSO, an inhibition halo greater than $15 \mathrm{~mm}$ was obtained for all the three species, indicating that all of them were susceptible to AmB-TFs. Halos $<10 \mathrm{~mm}$ indicate poor activity while an inhibition halos between 10 and $15 \mathrm{~mm}$ demonstrate that the efficacy of the formulation is dosedependent ${ }^{12}$. The lack of diffusion across the agar can result in poorer activity. AmB-TFs showed a good diffusivity capacity across the agar along with a good in vitro antifungal activity similar to AmB dissolved in DMSO.

\section{In vitro antiparasitic activity and cytotoxicity against macrophages}

L. (L.) amazonesis (MHOM/Br/79/Maria) and L. (V.) braziliensis (MHOM/Br/75/M2903) were selected to test the in vitro activity of the AmB-TFs formulation as these two species are responsible for most of the cutaneous leishmaniasis (CL) and mucocutaneous leishmaniasis (MCL) in Latin America ${ }^{13}$. The $\mathrm{IC}_{50}$ against the free form of the parasite (promastigotes) was $2.00 \pm 0.24$ and $3.42 \pm$ $0.15 \mu \mathrm{M}$ for L. (L.) amazonensis and L. (V.) braziliensis respectively, while higher $\operatorname{IC}_{50}(5.37 \pm 0.19$ and $7.08 \pm 0.34 \mu \mathrm{M}$ ) was observed for the intracellular forms (amastigotes) (Table S3 in SI). The $\mathrm{CC}_{50}$ against $\mathrm{J} 774$ macrophages was $33.98 \pm 4.02 \mu \mathrm{M}$ resulting in a SI of 6.3 and 4.8 for L. (L.) amazonensis and L. (V.) brazilensis, respectively. Compared to the AmB sodium deoxycholate micelles (Fungizone $^{\circledR}$ ), the AmB-TFs were 3-fold less toxic ${ }^{12}$.

\section{Histological studies}

Skin treated with AmB-TFs (Fig. 5a-b) or AmB-DMSO (Fig. 5c-d) showed major morphological changes, being more pronounced in the latter. In both cases, disorganization of connective tissue has been observed, which compromised the junction between the epidermis and the dermis, while as well as for the untreated skin, inflammatory cells were not observed. 

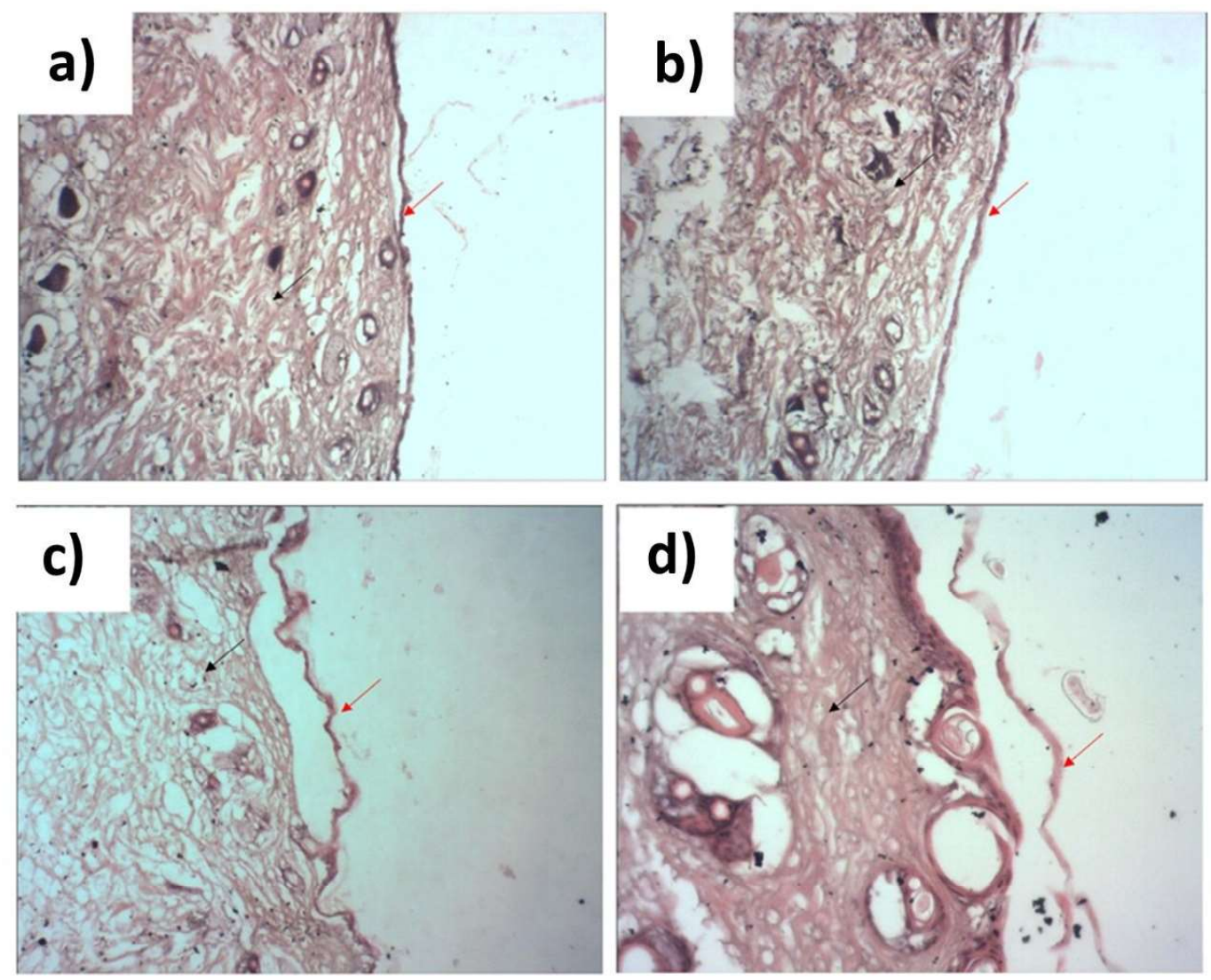

Figure 5. Micrographs of histological studies for treated skin with AmB -TFs and AmB dissolved in DMSO: a) mouse skin after exposure to AmB-TFs, 4x magnification; b) mouse skin after exposure to AmB-TFs, 10x magnification; c) mouse skin after exposure to AmB-DMSO, 4x magnification; d) mouse skin after exposure to AmB-DMSO; 10x magnification.

\section{In vivo topical pharmacokinetic studies}

In vivo tape striping was undertaken to access $\mathrm{AmB}$ levels within the $\mathrm{SC}$ and dermis after $0.5,1,2,4$ and 6 hours of exposure to AmB-TFs or the application of microneedles followed by topical administration of AmB-TFs immediately after which the animals were euthanised. As the thickness of the SC from the back of mice is approximately $15 \mu \mathrm{m}{ }^{14-16}$, the concentration in the SC $(15 \mu \mathrm{m} \sim 15$ tapes), dermis ( $\sim 12 \mu \mathrm{m})$ (Fig. 6) and remaining homogenised skin is summarised in Figure 7a. Selected time points for tape stripping were collected after steady state as established $\left(>1.7 \mathrm{t}_{\mathrm{lag}}\right){ }^{17}$. AmB encapsulated within TFs was able to permeate to deeper regions of the skin (Fig. 6a-b). Application of microneedles prior to the topical administration of AmB-TFs resulted in an increase in drug permeation during the first hour, especially in deeper regions of the skin. Application of solid microneedles resulted in transient micropores that facilitate drug delivery across the skin ${ }^{18}$, as confirmed by 0.5 and $1 \mathrm{~h}$ levels (Fig. 6c-d). However, micropores are known to reseal within $2 \mathrm{~h}$ when the skin is not occluded ${ }^{19,20}$ and the overall advantage is lost at later time points. This could also indicate the scar tissue formation limits 
AmB permeation as effective surface are for permeation has been reduced. At shorter times $(0.5 \mathrm{~h})$, AmB cumulative levels increase linearly, while over time, the permeation process was saturated. Diffusion is easier within the first 4-5 strips, while the diffusion is hindered in deeper skin areas as water osmotic gradient is less steep. Longer periods of AmB-TFs exposition to the skin resulted in greater amounts of drug accumulated in the skin (Fig. 7a). Bearing in mind the in vitro activity against amastigotes, effective concentrations are obtained after one hour from topical administration, and possibly even earlier $(0.5 \mathrm{~h})$ if microneedles are applied previous to topical application. However, the use of metallic microneedles did not result in statistical significant increase in $\mathrm{AmB}$ in the deeper skin layers at any time point, which means that transdermal delivery of $\mathrm{AmB}$ is unlikely (Figure 7a).

a)

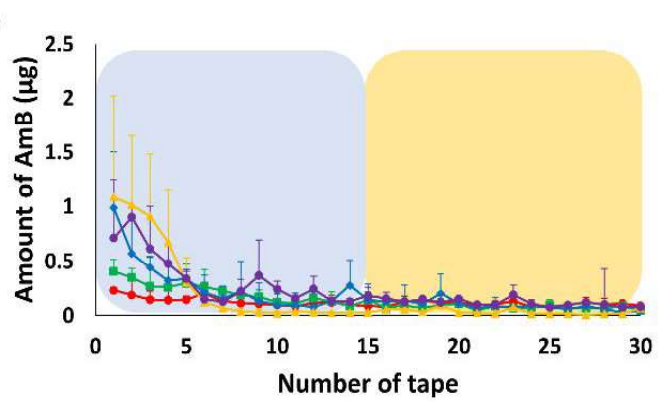

c)

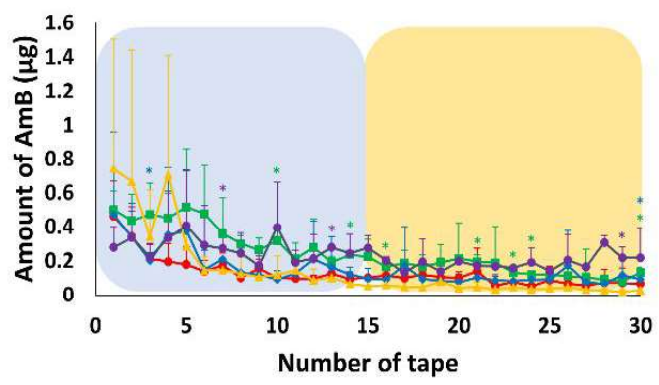

b)

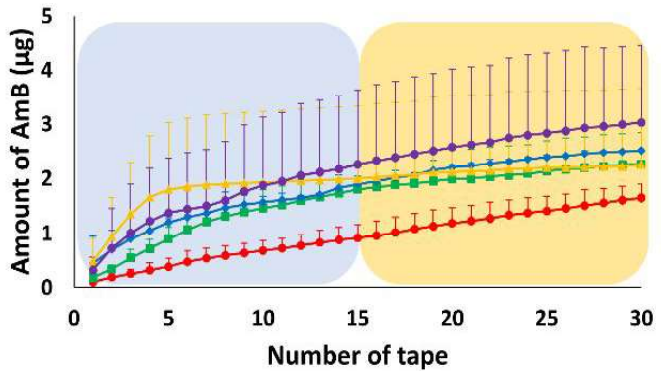

d)

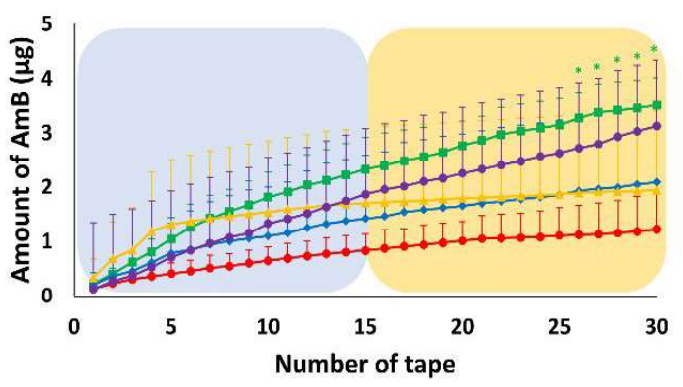

Figure 6. Averaged amount of $\mathrm{AmB}$ per tape strip (a, c) and cumulative amount of $\mathrm{AmB}(\mathrm{b}, \mathrm{d})$ in mouse skin after $0.5,1,2,4$ and $6 \mathrm{~h}$ of post-administration without the application of microneedles (a, b) and after the utilization of microneedles (c, d). Key: $0.5 \mathrm{~h}$ (red circles), $1 \mathrm{~h}$ (green squares), 2 $\mathrm{h}$ (blue diamonds), $4 \mathrm{~h}$ (yellow triangles), $6 \mathrm{~h}$ (purple circles). The blue and orange panels represent the stratum corneum and the evaluated skin once the stratum corneum is removed, respectively. Statistically significant differences are represented by * $(\mathrm{p}<0.05$, one way-ANOVA post-hoc Tukey’s test).
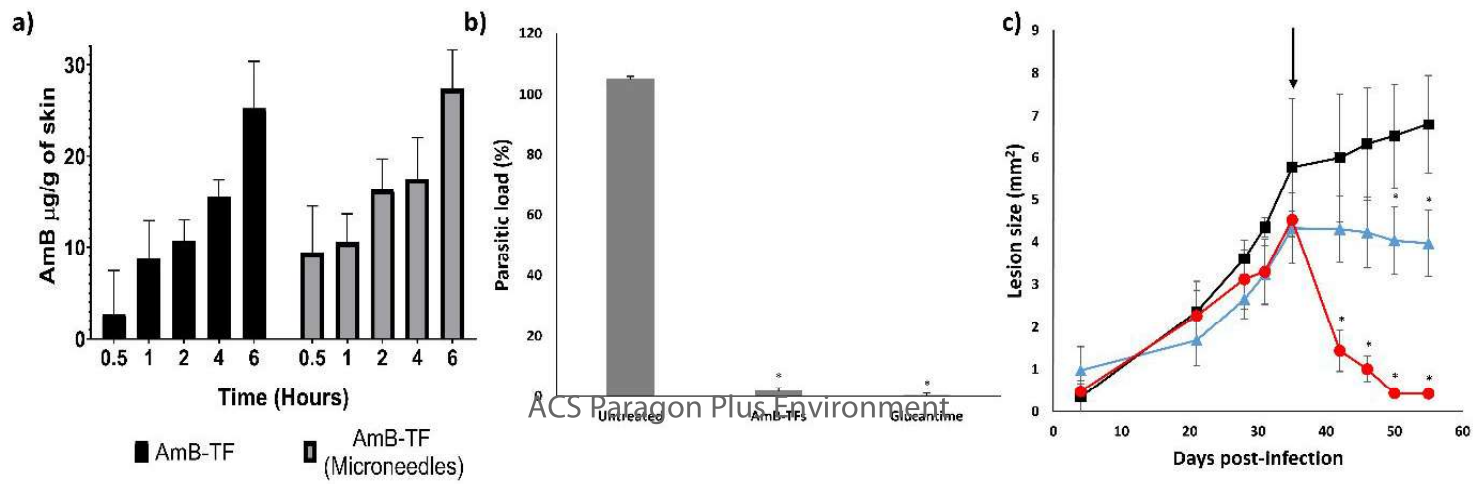
Figure 7. a) Amount of remaining AmB in the skin after removal of 30 tape-strips after the topical AmB-TFs administration (black bars) and topical AmB-TFs administration after application of microneedles (grey bars); b) In vivo antiparasitic activity (parasitic load) of AmB-TFs (0.5 mg/ml, $20 \mathrm{mg}$ of formulation/day, once daily topically applied) and Glucantime ${ }^{\circledR}(50 \mathrm{mg} / \mathrm{kg}$, once daily 25 $\mu$ l intralesionally administered) in experimental cutaneous leishmaniasis caused by $L$. (L.) amazonensis; c) Evaluation of lesion size during the course of the infection. Key: untreated animals (black squares), AmB-TFs (blue triangles), Glucantime ${ }^{\circledR}$ (red circles). Arrow indicates the onset of treatment. Statistically significant differences between treated and untreated animals is represented by * $(\mathrm{p}<0.05$, Mann-Whitney U test $)$.

\section{In vivo antiparasitic activity}

In vivo antiparasitic activity of topically administered AmB-TFs is illustrated in Figure 7b-c. The administration of topical AmB-TFs for 10 days resulted in a decrease in parasitic load of $98.24 \pm 1.54$ $\%$ similar to the decrease achieved with intralesionally administered Glucantime ${ }^{\circledR}(99.78 \pm 0.31 \%)$ with a p-value $>0.05$ (Fig. 7b, Table S4 in SI). When evaluating the lesion size, a decrease was observed for the Glucantime ${ }^{\circledR}$ and AmB- TFs from the sixth measurement (one month and a half post-infection and seven days after the beginning of the treatment). The untreated group showed a linear progression of the lesion size over time. The healing of the lesion was faster in animals intralesionally treated with Glucantime $^{\circledR}$, while in the case of AmB-TFs, the reduction in the lesion size was only significant at the eighth measurement (six days after the end of the treatment) (Fig. 7c). These results suggest that longer administration periods or potentially twice daily administration of topical AmB-TFs could lead to a total remission of the cutaneous lesion. From a practical point of view, AmB-TFs could be advantageous compared to Glucantime ${ }^{\circledR}$ as the latter is painful and requires intralesional administration in clinical settings limiting patient compliance, especially in developing countries ${ }^{21}$.

\section{DISCUSSION}

Here, we describe a topical AmB transfersomal formulation able to effectively deliver AmB across the stratum corneum and reach drug therapeutic levels in the epidermis to elicit a pharmacological effect as evidenced by in vivo pharmacokinetic and efficacy studies for the treatment of fungal and parasitic infections. For the latter, there is a strong need for topical therapies for CL and MCL, particularly for the milder and most common cases with up to 4 ulcers not exceeding $3 \mathrm{~cm}$ in diameter ${ }^{1}$. However, drug formulations for topical application have not met the expected impact. Paromomycin-based ointment marketed in the Middle East was not effective against New World parasite species ${ }^{22,23}$, while a cream containing $3 \%$ of AmB (Anfoleish) showed poor efficacy in a clinical study in Colombian CL patients 
${ }^{5}$ resulting in a range of adverse effects such as itching, redness, peeling dryness and irritation of the skin ${ }^{6}$. Thus, there is still an unmet clinical need for AmB topical formulations able to localise high amounts of $\mathrm{AmB}$ in the skin for fighting these infections.

DOE studies informed the design of the TFs in terms of drug loading, small particle size and colloidal stability prepared from generally regarded as safe excipients (GRAS). The choice of the edge activator is critical in the design of TFs and sodium deoxycholate was chosen as it is known to be a good solubiliser for AmB as well as a proven edge activator to ensure critical fluidity required for permeation of TFs ${ }^{8}$. Optimising the ratio between sodium deoxycholate and AmB was critical as there is an inverse relationship between the amount of sodium deoxycholate and particle size (Fig. 1). It is worth to highlight that sodium deoxycholate is the excipient traditionally utilised to solubilise $\mathrm{AmB}$ in Fungizone ${ }^{\circledR}$, in which dimeric $\mathrm{AmB}$ is formulated in a molar ratio 1:2 drug:excipient ${ }^{24}$. Although there are reports of skin irritation for $\mathrm{NaDC}$ when skin is exposed to this water soluble ionic surfactant, sodium deoxycholate stabilized transfersomes have not shown any signs of dermatological toxicity after topical application as supported by in vitro studies and clinical studies ${ }^{8,25}$.

Prepared AmB-TFs were able to solubilise high amounts of AmB comparable to those previously reported ${ }^{11}$. Sodium deoxycholate was also preferred over non-ionic surfactants, such as Tween ${ }^{\circledR} 80$ or $\operatorname{Span}^{\circledR} 80^{8,11,26,27}$ due to the greater EE obtained in preliminary studies. AmB-TFs exhibited an EE close to $95 \%$ which can be attributed to the combination of soya phosphatidylcholine and sodium deoxycholate $(86.87: 14.13 \mathrm{w}: \mathrm{w})$ which facilitates apolar interactions within the hydrophobic regions of both molecules as well as electrostatic interactions between the carboxylic group of the sodium deoxycholate and the primary amine of the AmB. The optimised formulation exhibited an AmB concentration of $0.086 \% \mathrm{w} / \mathrm{v}$. Higher drug concentrations could be achieved but resulted in drug precipitation and lower EE as well as vesicle aggregation. For this reason, existing drug loading was preferred to the expense of greater EE in order to ensure a small particle size $(\sim 150 \mathrm{~nm})$. TEM images confirmed quasi-spherical morphology of similar particle size with a clear bilayer surrounding an aqueous core.

One of the advantages of the optimised AmB-TFs is their enhanced physicochemical stability making their use feasible in developing countries without the need of cold transport chain. As TFs are typical aqueous suspensions, to prolong their stability, we also developed a lyophilised formulation. Freezedried AmB-TFs showed a good physicochemical stability ( $>90 \%$ drug content and particle size $<200$ $\mathrm{nm}$ ) over six months at 4 and $25{ }^{\circ} \mathrm{C}$ under desiccated conditions. Upon reconstitution, liquid AmB-TFs were stable over 30 days at $4{ }^{\circ} \mathrm{C}$ and at least over one week at $25^{\circ} \mathrm{C}$. This stability is superior than those reported in the marketed formulations, AmBisome ${ }^{\circledR}$ and Fungizone ${ }^{\circledR}$ (one day at $25{ }^{\circ} \mathrm{C}$ and one week under refrigerated conditions) ${ }^{28}$. 
In vitro permeation studies across Strat- $\mathrm{M}^{\circledR}$ membranes (synthetic ultrafiltration membranes composed of two layers of polyether sulfone on top of one layer of polyolefin) ${ }^{29-31}$ showed AmB-TFs had a lag time of $9.99 \pm 4.59$ minutes, while AmB-DMSO $4.86 \pm 2.87$ minutes. The steady state flux was 5.03fold lower for AmB-TFs. These membranes are constructed of two layers of polyether sulfone, resistant to diffusion, on top of one layer of polyolefin that is, open and diffusive. These polymers are able to create a porous structure with a gradient across the membrane in terms of pore size and diffusivity. The porous structure is impregnated with a proprietary blend of synthetic lipids, imparting additional skinlike properties. These membranes mimicking skin were previously shown for low molecular compounds with high lot-to-lot reproducibility ${ }^{30,31}$ and recently showed to have close similarity to human skin for high molecular weight drugs such as $\mathrm{AmB}^{29}$. However, across mouse skin, no statistical significant differences were found in the permeation rate across mouse skin between AmB-TFs and AmB-DMSO, presenting a similar steady-state flux during the first 4 hours as a result of the good permeability of the TFs across the skin. Flux was 6-fold and 4-fold higher than other AmB liposomal and transfersomal formulations, respectively, described previously across rat skin ${ }^{11}$. The latter transfersomal formulation contained soya phosphatidylcholine, cholesterol and $\mathrm{NaDC}$ in a molar ratio of 7:3:1. Flux was also similar to the flux across rat skin of AmB ethosomal preparations that contained a higher percentage of ethanol to the one included in our TFs ${ }^{29}$. However, AmB ethosomes exhibited a lag time across rat skin of 1.5 hours, while AmB-TFs did not show any lag time in biological skin, indicating that higher amount of ethanol would delay drug release ${ }^{29}$. This would be advantageous considering that an immediate release would provide a quicker permeation and, thus, a faster effect of the drug.

AmB-TFs illustrated in vitro efficacy in New World leishmaniasis species with comparable $\mathrm{IC}_{50}$ to previously studied ${ }^{32}$ and Candida spp. ${ }^{33}$. Higher concentrations of AmB were required to inhibit growth of amastigotes in comparison with promastigotes, bearing in mind that the first form of the parasite is located inside macrophages and hence, $\mathrm{AmB}$ molecules have to permeate the macrophage membrane in order to reach the parasite reservoir. However, it is key that the drug is selectively more active for parasites than for macrophages. $\mathrm{AmB}-\mathrm{TFs} \mathrm{IC}_{50}$ in amastigotes were higher than in previous reports ${ }^{34}$. Differences in origin of murine macrophages which could potentially result in differences in number of available LDL receptors could explain differences in uptake and thus activity, while effects of protein binding, aggregation state between them can potentially explain observed differences ${ }^{35,36}$. Additionally, lack of a clear water osmotic gradient in vitro could limit permeability in macrophages 2 , ${ }^{35-38}$. However, low in vitro $\mathrm{IC}_{50}$ values do not overcome challenges posed by the $\mathrm{SC}$ in vivo and in vitro previously tested results are not indicative of clinical scenario. TFs demonstrated a selectivity index towards amastigotes compared to mammalian macrophages that allows their clinical translation ${ }^{39}$. This is a therapeutic advantage considering that a limited toxicity allows a higher drug administration dose and hence, higher chance to eradicate the parasites in a multiple dose regime. Entrapping AmB in TFs 
reduced the haemolytic effects of $\mathrm{AmB}$, while after 6 hours of skin exposure little if any inflammation was histologically observed. No signs of toxicity was also visible after in vivo application of AmB-TFs in infected mice.

In our study, in vivo topical bioavailability of the formulation in mice was also evaluated. To the best of our knowledge, this is the first time that the pharmacokinetic profile of AmB loaded in TFs is studied. AmB encapsulated in TFs was able to cross the stratum corneum and reach deeper regions of the skin at concentrations above the $\mathrm{IC}_{50}$ for $L$. (L.) amazonesis and $L$. (V.) braziliensis, but also below the $\mathrm{CC}_{50}$ for macrophages. From a clinical point of view, this is especially advantageous bearing in mind that parasites are located in the epidermis and dermis ${ }^{40}$. The use of microneedles prior the administration of AmB-TFs resulted in enhanced drug permeability in SC and dermis up to two hours postadministration. This was in good agreement with studies by other authors under non-occlusive conditions which show that the formed pores heal within two hours, while healing is delayed for up to several days depending on the geometry of the applied microneedles under occlusion ${ }^{14,41,42}$.

The efficacy of AmB-TFs was also demonstrated in vivo, where the parasitic load was reduced greater than $98 \%$ after once a day administration for 10 days, exhibiting the same efficacy (p-value $<0.05$ ) as intralesionally administered antimonials. The use of microneedles prior to the AmB-TFs topical administration enhanced even further the $\mathrm{AmB}$ permeation during the first hour post-application. Previous studies have demonstrated that drug permeability is enhanced in Leishmania-infected skin ${ }^{11}$. The high efficacy demonstrated by AmB-TFs could also be explained by the fact that trans-epidermal water loss (TEWL) is usually around 2.5-fold higher on skin infected with Leishmania spp. ${ }^{43}$ and is associated to damaged epidermal and dermal skin layers and reduced density of collagen and effectiveness of the barrier function of skin ${ }^{44}$. As TFs permeation is driven by the water osmotic gradient and Leishmania infected skin is dryer, it is likely that the gradient for TFs permeation is steeper in infected animals and potentially patients. This can potentially explain the enhanced efficacy of our AmB-TFs formulation across infected skin.

Two alternative AmB-TFs semisolid formulations were also prepared by mixing at 1:1 w:w the AmBTFs with different gelling agents, Carbopol ${ }^{\circledR} 940$ (at $8 \%$ ) and Metolose $^{\circledR} 1500$ (combination of methylcellulose and hypromellose at $4 \%{ }^{45}$. However, the in vitro permeation across the skin was limited compared to the liquid formulation probably related to a hindered released from the gel (data not shown). AmB ethosomal gel (1\% w/w) prepared with Carpobol $^{\circledR} 934(1.5 \% \mathrm{w} / \mathrm{w})$ have been shown to maintain a high flux in previous reports ${ }^{29}$. Further studies are needed to establish the concentration of the gelling agent needed for optimal permeability. The intralesional administration of the AmB-TFs is also feasible if required in severe cases of CL. A liquid formulation would be also beneficial from patients suffering from fungal skin infections disseminated along larger regions of the body.

\section{CONCLUSIONS}


Our AmB-TFs showed enhanced in vitro permeation across Strat-M ${ }^{\circledR}$ and mouse skin and elicited therapeutic levels after in vivo administration as confirmed by tape stripping and reduction in parasite number in infected mice. Our formulations prepared from GRAS excipients showed improved safety according to haemolysis, cytotoxicity and histological studies and are thermally stable without the need for a cold transport chain. This formulation is readily translatable as a good therapeutic alternative to current treatments for CL and fungal infections in developed and developing countries.

\section{METHODS}

\section{Materials}

AmB ( $\geq 98 \%)$ was procured from North China Pharmaceutical Huasheng Co (Hebei, China). Phosphatidylcholine (Lipoid $\mathrm{S} 100^{\circledR}$ ) was donated from Lipoid GmbH (Ludwigshafen, Germany). Sodium deoxycholate, other chemicals and solvents, as well as Roswell Park Memorial Institute 1640 medium (RPMI-1640) utilized in the culturing of Leishmania parasites, were obtained from SigmaAldrich Chemie GmbH (Madrid, Spain) and were at least ACS grade. The rest of the materials were purchased as follows: ethanol $96^{\circ}$ (Alcoholes Aroca S.L., Madrid, Spain), Strat-M ${ }^{\circledR}$ membranes (Merck KGaA, Darmstadt, Germany), Triton $\mathrm{X}^{\circledR}-100$ (Sigma-Aldrich Co, St. Louis, USA), Sabouraud dextrose agar (Becton, Dickinson and Co., New Jersey, USA), Müller-Hinton agar (Laboratorios Conda S.A., Madrid, Spain), glucose (Panreac Química S.A.U., Barcelona, Spain) and sterile 0.9 \% sodium chloride (Laboratorios ERN, S.A., Barcelona, Spain)

\section{Design of experiment (DOE) of AmB-TFs}

A three-level Box-Behnken model was selected to obtain an optimised TFs formulation. DesignExpert ${ }^{\circledR}$ 10 software (Stat-Ease Inc., Minneapolis, USA) was utilised to establish the high and low levels of the design. The concentration of edge activator (sodium deoxycholate), the amount of AmB and the percentage of ethanol used to reconstitute the dry film were the chosen factors (independent variables), while encapsulation efficiency (EE), particle size and zeta potential were the selected responses (dependent variables) for optimisation. Ranges of each factor are described in Table 3. Seventeen formulations were prepared using different combinations of the factors. The batch size was kept constant (200 mg in $2 \mathrm{~mL}$ ). The amount of AmB and edge activator was added based on the DOE (Table 2) and the amount of lipid was calculated as the remaining till $200 \mathrm{mg}$ total amount.

Table 3. Selected factors and levels to implement the Box-Behnken design of experiments for the optimisation of the AmB-TFs formulation.

\begin{tabular}{|c|c|c|c|}
\hline \multirow{2}{*}{ Factor } & Low & Medium & High \\
\cline { 2 - 4 } & 6 & 13 & 20 \\
\hline $\begin{array}{c}\text { Concentration of edge } \\
\text { activator (\%) }\end{array}$ & 0.5 & 1.25 & 2 \\
\hline $\begin{array}{c}\text { Amount of drug (mg) } \\
\text { per 2 ml batch }\end{array}$ & 0 & \\
\hline
\end{tabular}


Percentage of ethanol in the reconstitution buffer (\%)

Other variables involved in the manufacturing process were kept constant through the 17 runs such as: rotaevaporation time $(30 \mathrm{~min})$, reconstitution volume $(2 \mathrm{~mL})$ and probe sonication time.

\section{Optimisation of AmB-TFs formulation and validation of the model}

Mathematical modelling was carried out by multiple linear regression analysis (MLRA). Only the statistically significant coefficients $(\mathrm{p}<0.05)$ were considered in framing the polynomial equations, and the model was evaluated by analysing the p-value and the determination coefficient $\left(\mathrm{R}^{2}\right)$. Response surface analysis was carried out employing 2D and 3D plots for understanding the relationship among the amount of edge activator, drug and ethanol on particle size and EE. The prognosis of the optimum formulation was conducted by numerical optimization and desirability function with the aim of maximising EE and particle size ranging from 100 to $300 \mathrm{~nm}$. Validation of the QbD methodology was performed by comparing the predicted responses with the experimental ones.

\section{Preparation of AmB-TFs}

AmB-TFs were prepared by the thin film hydration method. AmB (1.82 mg), phosphatidylcholine Lipoid $\mathrm{S}_{100}{ }^{\circledR}(171.74 \mathrm{mg})$ and $\mathrm{NaDC}(28.26 \mathrm{mg})$ were dissolved in methanol $(20 \mathrm{~mL})$ in a roundbottom flask. Solvent was evaporated protected from light under vacuum using a rotaevaporator RV 06-ML (Janke \& Kunkel Ika-Labortechnik, Staufen, Germany) and a vacuum pump Vac ${ }^{\circledR}$ V-500 (Büchi Labortechnik, Flawil, Switzerland) controlled by a vacuum controller B-721 (Büchi Labortechnik, Flawil, Switzerland) till a dry film was obtained at $43{ }^{\circ} \mathrm{C}$ and 250 r.p.m.. The film was hydrated by adding $2 \mathrm{~mL}$ of a mixture of phosphate buffered saline (PBS) pH $7.4(295.8 \mathrm{mOsm} / \mathrm{L})$ and ethanol $(91.5: 8.5 \mathrm{v}: \mathrm{v})$ under rotation using the rotaevaporator over 30 minutes while being protected from light. The resulting suspension was then bath sonicated (150 Watts, P Selecta Ultrasons-H, Barcelona, Spain) for 30 minutes and probe sonicated (10 Watts, two cycles of 10 seconds with 50 seconds in between to avoid overheating, Branson Sonifier 250, Emerson Industrial, Connecticut, USA). The formulation was centrifuged at room temperature $(6,000 \mathrm{rpm}, 15 \mathrm{~min})$ using a Universal $32^{\circledR}$ centrifuge (Hettich Zentrifugen, Tuttlingen, Germany) to separate the unencapsulated AmB.

\section{Lyophilisation of optimised AmB-TFs formulation}

Mannitol was added to the liquid optimised AmB-TFs formulation as a cryoprotectant. Mixtures were frozen at $-40{ }^{\circ} \mathrm{C}$ overnight and freeze-dried at $-50{ }^{\circ} \mathrm{C}$ and a pressure of 0.2 bar for 24 hours in a LyoQuest freeze-drier (Azbil Telstar, S.L., Terrassa, Spain). The final solid concentration in the lyophilised formulation was $11.6 \% \mathrm{w} / \mathrm{v}$, containing $0.923 \mathrm{mg}$ of $\mathrm{AmB}, 173.1 \mathrm{mg}$ of phosphatidylcholine, $26 \mathrm{mg}$ of sodium deoxycholate and $33.35 \mathrm{mg}$ of mannitol. 


\section{Characterisation of the optimised AmB-TFs}

Particle size and zeta potential were measured in freshly prepared AmB-TFs using a Zetatrac Ultra (Microtrac Inc, Pennsylvania, USA) analyser. Particle size was measured by dynamic light scattering (DLS). Prior to measurements, samples were diluted with deionised water $(1 / 100 \mathrm{v} / \mathrm{v})$.

Morphology of the optimised formulation was evaluated using transmission electron microscopy (TEM) (S2.1 in SI). EE was measured after centrifugation at 6,000 rpm for $15 \mathrm{~min}$ in a Universal $32^{\circledR}$ centrifuge (Hettich Zentrifugen, Tuttlingen, Germany) at room temperature. The supernatant was diluted with methanol $(1 / 100, v / v)$. The absorbance was measured using a PharmaSpec ${ }^{\circledR}$ UV-visible spectrophotometer (Shimadzu, Kyoto, Japan) between 300 and $450 \mathrm{~nm}$ wavelength to ensure all the drug was in the monomeric state ${ }^{1}$. The absorbance at $406 \mathrm{~nm}$ was recorded. EE was calculated using the following equation ${ }^{46}$ (Eq. 4):

$$
\left.E E(\%)=\frac{A-B}{A} \times 100 \quad \text { (Eq. } 4\right)
$$

where A was the absorbance at $406 \mathrm{~nm}$ of the formulation after probe sonication before centrifugation and $\mathrm{B}$ was the absorbance at $406 \mathrm{~nm}$ of the formulation after centrifugation.

\section{Physicochemical stability of AmB-TFs}

Freshly prepared AmB-TFs were diluted with PBS pH $7.4313 \mathrm{mOsm} / \mathrm{L}(1 / 15 \mathrm{v} / \mathrm{v})$, consisting on $\mathrm{NaCl}$ $(8 \mathrm{~g} / \mathrm{L}), \mathrm{KCl}(0.2 \mathrm{~g} / \mathrm{L}), \mathrm{Na}_{2} \mathrm{HPO}_{4}(1.44 \mathrm{~g} / \mathrm{L})$ and $\mathrm{KH}_{2} \mathrm{PO}_{4}(0.24 \mathrm{~g} / \mathrm{L})$. The batch was then divided in different vials containing $1 \mathrm{~mL}\left(79.8 \mu \mathrm{g}\right.$ of $\left.\mathrm{AmB} \mathrm{mL}^{-1}\right)$ each. Half of the vials were lyophilised as described above. Both formulations were stored at 4 or $25{ }^{\circ} \mathrm{C}$. The effect of relative humidity (RH) on the stability of lyophilised formulation was assessed at $25^{\circ} \mathrm{C}$ that was controlled at 10 and $60 \%$ using different saturated salt solutions ${ }^{47}$. Lyophilised formulations were placed in uncapped vials. Prior to analysis, freeze dried samples were reconstituted with $1 \mathrm{ml}$ of deionised water. At different time points, samples were withdrawn and particle size and drug content by HPLC ${ }^{48}$ were measured.

\section{In vitro permeability studies}

Male BALB/c untreated mice ( 8 weeks old, 26-28g, Harlan, UK) were euthanised and hair was trimmed prior skin being freshly excised and frozen in $-50^{\circ} \mathrm{C}$ in flat closed plastic bags. The skin was thawed and shaved, then suspended in $60^{\circ} \mathrm{C}$ deionised water for 30 seconds to manually remove the underlying muscle tissue and hypodermis ${ }^{14}$. Then the skin was placed flat in plastic bags and stored in $-50{ }^{\circ} \mathrm{C}$ freezer till further use. When needed, the skin was thawed in receptor fluid buffer prewarmed at $37^{\circ} \mathrm{C}$ prior use. Strat- $\mathrm{M}^{\circledR}$ membranes required no preparation.

Diffusion studies of optimised AmB-TFs (0.086\% w/v) and equivalent AmB in dimethylsulfoxide (DMSO) at $0.086 \% \mathrm{w} / \mathrm{v}$ were performed using vertical diffusion Franz Cells (Soham Scientific, 
Loughborough, UK). The diffusion diameter of each Franz cell was measured using a caliper $( \pm 0.01$ $\mathrm{mm}$ ) and the surface area ranged between $5.29-5.34 \mathrm{~cm}^{2}$. Strat- ${ }^{\circledR}$ membranes (Merck KGaA, Darmstadt, Germany) or mouse skin were placed on receptor compartment with the stratum corneum facing upwards and a stirring bar $(3 \times 5 \mathrm{~mm})$ was added to each Franz Cell's receptor compartment and then filled completely with $4 \% \mathrm{w} / \mathrm{v}$ sodium deoxycholate in PBS (pH 7.4) to ensure sink conditions. Strat- $\mathrm{M}^{\circledR}$ membranes (synthetic models for transdermal diffusion testing) required no preparation according to the recommendations of the manufacturer ${ }^{49}$. The donor compartment, the membrane or skin, and the receptor compartment were tightly sealed using ParaFilm ${ }^{\mathrm{TM}}$ and clamped together. PBS $(1 \mathrm{~mL})$ was added into the donor compartment and then placed into a water bath at $37{ }^{\circ} \mathrm{C}$ (Tempette Junior TE-8J, Techne, Staffordshire, UK) for 30 minutes under magnetic stirring (Fisherbrand, Leicestershire, UK). After 30 minutes, the PBS and receptor media were removed for analysis and the receptor compartment filled with fresh and prewarmed $4 \% \mathrm{w} / \mathrm{v}$ sodium deoxycholate in PBS (pH 7.4). AmB-TFs or AmB in DMSO (0.6 g of each formulation at an AmB concentration of $0.086 \% \mathrm{w} / \mathrm{v}$ equivalent to an $\mathrm{AmB}$ dose utilized of $0.517 \mathrm{mg}$ ) were added to the donor chamber. At selected time points $(0,20,40,60,90,120,150,180,240,300$ and $360 \mathrm{~min}), 0.5 \mathrm{ml}$ of the receptor medium was taken using a syringe and immediately refilled with $0.5 \mathrm{ml}$ of fresh media. AmB concentration was quantified in the samples using a validated HPLC method ${ }^{48}$ (Jasco Inc., Maryland, USA) with a lower limit of detection (LoD) of $0.19 \mu \mathrm{g} \mathrm{mL}^{-1}$.

The cumulative amounts of $\mathrm{AmB}$ permeated through the Strat- $\mathrm{M}^{\circledR}$ membrane and the mouse skin were plotted as a function of time ${ }^{50}$. Regression analysis was used to calculate from the slopes and intercepts of the linear portion of each graph. The following equation (Eq. 5) was applied to each formulation to calculate the steady-state flux:

$$
J s S=\frac{d C}{d X} * \frac{1}{A}
$$

where Jss is the steady-state flux $\left(\mu \mathrm{g} / \mathrm{cm}^{2} / \mathrm{h}\right), \mathrm{dC} / \mathrm{dX}$ is the amount of AmB permeating the membrane over time $(\mu \mathrm{g} / \mathrm{h})$ and $\mathrm{A}$ is the surface area of contact of the formulation ${ }^{51}$. The permeability coefficient (P) was calculated by using equation 6:

$$
P=\frac{J s s}{c d}
$$

where cd is the amount of drug applied in donor compartment $(0.6 \mathrm{~g}$ of formulation equivalent to 0.517 $\mathrm{mg}$ of $\mathrm{AmB}$ ). Diffusion coefficient was calculated by using the following equation:

$$
D=P h
$$

where $\mathrm{h}$ is the thickness of the membrane $(0.62 \pm 0.08 \mathrm{~mm})^{52}$. 
After permeability studies with mouse skin, skin was cleaned with a cotton bud to remove excess of formulation. Samples were cut in two halves. One half was fixed using $4 \%$ paraformaldehyde (pH 7). In the other half, AmB was extracted ( 3 times) using $3 \mathrm{ml}$ of buffer with $4 \%$ sodium deoxycholate: methanol (1:1 v:v). After each extraction, the homogenate was vortexed ( $2 \mathrm{~min})$ and centrifuged $(8,000$ rpm, $10 \mathrm{~min}$, room temperature) and supernatant was collected and transferred into HPLC vials. The supernatant was analysed by a validated HPLC method ${ }^{48}$. Statistical analysis was performed by 2-tail, unequal variance, student t-test on flux at each time point.

\section{Histological studies}

Mouse skin samples exposed to AmB-TFs, AmB-DMSO for 6 hours in the Franz Cells, as well as pretreated skin that had not been exposed to any formulation at all were fixed separately with paraformaldehyde $(4 \%, \mathrm{pH} 7.4)$ and refrigerated $\left(2-8{ }^{\circ} \mathrm{C}\right)$. Histological studies were performed as previously described (S2.2 in SI) ${ }^{14,50,53}$.

\section{Ex vivo red blood cells haemolysis assay}

Ex vivo haemolytic activity of $\mathrm{AmB}$ was determined using blood taken from a healthy human donor (female, 32 years old, haematocrit: $45 \%$ ) as previously described with few modifications (S2.3 in SI) ${ }^{54}$. RBCs $(100 \mu \mathrm{L}, 4 \% \mathrm{w} / \mathrm{v})$ were added to a 96 -well plate followed by AmB-TFs $(100 \mu \mathrm{L})$ diluted to various concentrations between 270 to $4 \mu \mathrm{M}$. AmB-unloaded TFs in PBS were diluted at the same concentrations and were also tested. PBS (pH 7.4) and Triton $\mathrm{X}^{\circledR}-100$ diluted to $20 \%$ in PBS (pH 7.4) were used as negative and positive controls respectively. AmB dissolved in DMSO was also used as a control. Haemolysis at each concentration was calculated by using the following equation (Eq. 8):

$$
\text { Haemolysis }(\%)=\frac{A b s(s)-A b s(P B S)}{A b s(\text { Triton })-A b s(P B S)} \times 100
$$

where $\mathrm{Abs}(\mathrm{s})$ is the average absorbance of the sample at each concentration tested, Abs (PBS) is the average absorbance of the negative control, and Abs (Triton) is the average absorbance of the positive control. Data were analysed using Compusyn ${ }^{\circledR}$ (Combosyn Inc, New Jersey, USA) software and $\mathrm{HC}_{50}$ (concentration needed to haemolyse $50 \%$ of erythrocytes) was obtained.

\section{In vitro antifungal activity}

The in vitro antifungal activity of $\mathrm{AmB}$ was determined according to European Pharmacopeia standards in three different species of Candida spp.: C. albicans, C. glabrata and C. parapsilosis (S2.4 in SI) ${ }^{12}$. One-way ANOVA was undertaken using Minitab ${ }^{\circledR} 16$ software.

\section{In vitro antiparasitic activity}

Two species of Leishmania spp., L. (L.) amazonensis and L. (V.) braziliensis were cultured in Schneider's insect medium (Sigma-Aldrich Co, St. Louis, USA) at $26{ }^{\circ} \mathrm{C}$ supplemented with $20 \%$ heat- 
inactivated foetal bovine serum (FBS) (Sigma-Aldrich Co, St. Louis, USA), penicillin (100 IU/mL) and streptomycin $(100 \mu \mathrm{g} / \mathrm{mL})$ in culture flasks $(25 \mathrm{~mL}){ }^{55,56}$. Two different assays against promastigotes and amastigotes were carried out with resazurin, following methods previously described in literature (S2.5 in SI) ${ }^{56}$. The efficacy of each compound was determined by calculating the $\mathrm{IC}_{50}$ (concentration of formulation needed to inhibit $50 \%$ growth) ${ }^{56}$.

\section{In vitro macrophage cytotoxicity}

Macrophage cytotoxicity was determined in J774 murine macrophages (S2.6 in SI) ${ }^{55,56}$. The results were expressed as $\mathrm{CC}_{50}$ (concentration that produced cytotoxicity in $50 \%$ of macrophages). Selectivity index (SI) was calculated to evaluate the relationship between efficacy and toxicity for AmB-TFs using the following equation (Eq. 9):

$$
S I=\frac{C C_{50}}{I C_{50}}
$$

\section{Animals for efficacy and topical pharmacokinetics studies}

Mice were obtained from the Medical School of University of São Paulo, Brazil. The studies were carried out in accordance with the recommendations detailed in the Guide of the Care and Use of Laboratory Animals of the Brazilian National Council of Animal Experimentation (http://www.cobea.org.br). The protocol was approved by the Ethics Committee of Animal Experiments of the Institutional Committee of Animal Care and Use at the Medical School of the University of São Paulo (CEUA 056/16). Mice were housed according to the standards of the Committee of Animal Welfare and allowed access to food and water ad libitum throughout the study under a $12 \mathrm{~h}$ light cycle. The animals were anesthetized with intraperitoneal sodium thiopental $(1 \mathrm{mg} / 200 \mu \mathrm{L})$.

\section{Efficacy studies in $L$. (L.) amazonensis infected mice}

L. (L.) amazonensis (MHOM/BR/73/M2269) was kindly provided by Prof. Dr. Fernando T. Silveira from the cryobank of the "Leishmaniasis Laboratory Prof. Dr. Ralph Laison", Department of Parasitology, Ministry of Health, Evandro Chagas Institute (Belém, Pará - Brazil). Species were identified using monoclonal antibodies and isozyme electrophoretic profiles at the Leishmaniasis Laboratory of the Evandro Chagas Institute in late log stage were used. Parasites were maintained in Schneider's medium (Sigma-Aldrich Co., St. Louis, USA) supplemented with 10 \% FBS, 50,000 IU/mL penicillin and $50 \mu \mathrm{g} / \mathrm{mL}$ streptomycin.

BALB/c mice (male, 5-weeks old) were randomly divided in four groups of four animals per group. Animals were inoculated at the base of the tail with $2 \times 10^{7}$ promastigote forms of $L$. (L.) amazonensis in stationary growth phase (final volume $25 \mu \mathrm{L}$ ), except for the healthy control group which was given only physiological solution $(25 \mu \mathrm{L})$. After 35 days of infection, treatment was started for 10 consecutive days applied once daily. Animals were arranged as follows: group 1 was treated with AmB-TFs (0.5 
$\mathrm{mg} / \mathrm{mL}, 20 \mathrm{mg}$ of formulation/day, topically applied), group 2 was treated with Glucantime ${ }^{\circledR}(50 \mathrm{mg} / \mathrm{kg}$, $25 \mu \mathrm{L}$ intralesionally injected), group 3 was the infected control group with no treatment and group 4 consisted on untreated and uninfected animals (healthy control group). Lesion size was monitored at days $4,21,28,31,35,42,46,51$ and 56 post-infection. Skin parasitism was quantified 10 days after the end of the treatment (at 56 days post-infection). The same animals were used to measure the lesion size and the parasite load.

The parasitic load was quantified by limiting dilution analysis in fragments of the skin, at the point of the parasite inoculation. Briefly, fragments of skin were collected and aseptically homogenized in Schneider's medium. Skin suspensions were subjected to 12 serial dilutions with four replicate wells. The number of viable parasites was determined based on the highest dilution that promastigotes could grow after 10 days of incubation at $25{ }^{\circ} \mathrm{C}$. In vivo experiments were repeated at least twice, and the results were expressed by the arithmetic mean \pm standard deviation. Statistical analyses were performed using GraphPad Prism 5.0, and the nonparametric Mann-Whitney U test was used to evaluate the differences between treated and infected control groups. Differences were considered statistically significant at a $5 \%$ significance level $(\mathrm{p}<0.05)$.

\section{Topical pharmacokinetic studies}

CD-1 male mice ( 8 weeks old, $28 \pm 2$ g) were randomly split into two different groups $(n=3)$ that received topically AmB-TFs with or without the application of microneedles for 3 seconds (Derma Pen with 12 metallic needles of $500 \mu \mathrm{m}$ in length purchased from TBPHP, Ruboskizo, Madrid, Spain). Prior to sample administration, hair was trimmed in the upper dorsal area of the body. A $1 \mathrm{~cm}^{2}$ of internal diameter plastic tube was adhered on the shaved area and $0.5 \mathrm{~mL}$ of AmB-TFs $\left(0.86 \mathrm{mg} \mathrm{mL}^{-1}\right)$ was applied. At different time points $(0.5,1,2,4$ and 6 hours post-administration), animals were sacrificed and subsequently, skin was removed and the area exposed to the formulation was cleaned with wet cotton bud before storage at $-20{ }^{\circ} \mathrm{C}$ prior to $\mathrm{AmB}$ analysis.

Skin samples were analysed by tape-stripping. Thirty consecutive tape-strips of $1 \mathrm{~cm}^{2}$ diameter (Punts ${ }^{\circledR}$, Etyprinter S.L., Barcelona, Spain) were adhered in the skin area where the formulation was deposited under the application of a $100 \mathrm{~g}$ weight for 2 seconds. Strips were removed and individually introduced into a plastic Eppendorf tube containing methanol $(0.45 \mathrm{~mL})$. Tubes were sonicated for $15 \mathrm{~min}$ at room temperature and centrifuged (5,000 rpm for $5 \mathrm{~min}$ at room temperature). The supernatant was removed and transferred to HPLC vials for further analysis using a validated method for AmB ${ }^{48}$. After the removal of the 30 layers, the remaining skin was weighted prior to being homogenised in a $1 \mathrm{~mL}$ of PBS pH 7.4 using a mortar and pestle. Two extractions using methanol were performed (1 $\mathrm{mL} \times 2)$. After each extraction, the homogenate was vortexed ( $2 \mathrm{~min})$ and centrifuged (8,000 rpm, $10 \mathrm{~min})$ and supernatant was collected and transferred into HPLC vials to be analysed as described above ${ }^{48}$. One- 
way ANOVA followed by a Tukey's post-hoc test was performed (Minitab ${ }^{\circledR} 16$ software) and differences were considered statistically significant at a $5 \%$ significance level $(\mathrm{p}<0.05)$.

\section{ASSOCIATED CONTENT SUPPORTING INFORMATION}

The Supporting Information is available free of charge. Detailed methodology on the characterisation of AmB transfersomes is provided (particle size, zeta-potential, encapsulation efficiency, morphological studies) as well as detailed methodology regarding histology studies and an in vitro antifungal efficacy, antiparasitic efficacy and macrophage cytotoxicity. Results regarding DOE studies for AmB transfersomes (Figure S1-S7, Table S1 and S2) as well as in vitro antiparasitic efficacy and macrophage cytotoxicity data (Table S3) are provided. Parasitic load measured in BALB/c mice treated with various formulations are also summarised (Table S4).

\section{AUTHOR CONTRIBUTIONS}

Raquel Fernández-García ${ }^{1 \#:}$ : Methodology (Formulation development, stability studies, morphology studies, in vitro antifungal studies, haemolysis studies, in vivo pharmacokinetic studies), Formal analysis, Investigation, Data Curation, Writing - Original Draft, Visualisation, Writing - Review \& Editing.

Larry Statts ${ }^{2 \#}$ : Methodology (Formulation development, DOE studies, Franz cells studies and histology studies), Validation, Formal analysis, Investigation, Data Curation, Formal analysis, Writing - Original Draft, Visualisation.

Jéssica A. de Jesus 3 : Methodology (In vivo efficacy studies), Validation, Formal analysis, Investigation, Data Curation, Formal analysis, Writing - Original Draft.

M. Auxiliadora Dea-Ayuela 4 : Methodology (In vitro antileishmanial and macrophage cytotoxicity studies), Validation, Formal analysis, Investigation, Data Curation, Formal analysis, Writing - Original Draft.

Liliana Bautista ${ }^{2}$ : Methodology (Formulation development and morphology studies), Formal analysis, Investigation, Data Curation.

Rúben Simãoㄹ: Methodology (In vivo pharmacokinetic studies), Formal analysis, Investigation.

Francisco Bolás-Fernández 5 : Supervision, Funding acquisition.

M. Paloma Ballesteros ${ }^{1,6}$ : Supervision, Review \& Editing.

Marcia Dalastra Laurenti ${ }^{3}$ Resources, Data Curation, Writing - Original Draft, Supervision, Project administration and execution, Funding acquisition 
Luiz F. D. Passero ${ }^{7,8}$ : Methodology (In vivo efficacy studies), Validation, Formal analysis, Investigation, Resources, Data Curation, Writing - Original Draft, Supervision, Project administration and execution, Funding acquisition

Aikaterini Lalatsa ${ }^{2 *}$ : Conceptualisation, Methodology (Formulation development, morphology studies and histology studies), Validation, Formal analysis, Investigation, Resources, Data Analysis and Curation, Writing - Original Draft, Visualisation, Supervision, Writing - Review \& Editing, Project administration and execution, Funding acquisition

Dolores R. Serrano ${ }^{1,6^{*}}$ : Conceptualisation, Methodology (Formulation development, DOE studies, Franz cells studies, in vitro antifungal efficacy, haemolysis studies, in vivo pharmacokinetic studies), Validation, Formal analysis, Investigation, Resources, Data Curation, Writing - Original Draft, Visualisation, Writing - Review \& Editing, Supervision, Project administration and execution, Funding acquisition

\title{
ACKNOWLEDGEMENTS
}

The authors are grateful to FAPESP (2016/0048-0) and HC-LIM50. Larry Statts was supported with an Erasmus $^{+}$grant to undertake a placement at UCM, Madrid, Spain. This work was funded by an IberoAmerican Universities Union Research Collaboration Fund (Unión Iberoamericana de Universidades; ENF03-2017) allowing student mobility exchange between UCM (Madrid, Spain) and USP (Sao Paulo, Brazil).

\section{CONFLICT OF INTERESTS}

The authors declare no competing financial interest.

\begin{abstract}
ABBREVIATIONS
AmB: Amphotericin B; AmB-TFs: Amphotericin B loaded trasnfersomes; DOE: Design of experiment studies; DMSO: Dimethylsulfoxide; EE: Entrapment efficiency; HPLC: High performance liquid chromatography; NaDC: Sodium deoxycholate; QbD: Quality by design; PBS: Phosphate buffered saline; RBCs: Red blood cells; RH: Relative humidity; SC: Stratum corneum; TEM: Transmission electron microscopy; TFs: Transfersomes
\end{abstract}

\section{REFERENCES}

1. Fernandez-Garcia, R.; de Pablo, E.; Ballesteros, M. P.; Serrano, D. R., Unmet clinical needs in the treatment of systemic fungal infections: The role of amphotericin $B$ and drug targeting. Int J Pharm 2017, 525 (1), 139-148.

2. Serrano, D. R.; Hernandez, L.; Fleire, L.; Gonzalez-Alvarez, I.; Montoya, A.; Ballesteros, M. P.; Dea-Ayuela, M. A.; Miro, G.; Bolas-Fernandez, F.; Torrado, J. J., Hemolytic and pharmacokinetic studies of liposomal and particulate amphotericin B formulations. Int J Pharm 2013, 447 (1-2), 38-46. 3. Torrado, J. J.; Espada, R.; Ballesteros, M. P.; Torrado-Santiago, S., Amphotericin B Formulations and Drug Targeting. J Pharm Sci 2008, 97 (7), 2405-2425. 
4. Jensen, G. M., The care and feeding of a commercial liposomal product: liposomal amphotericin B (AmBisome ${ }^{\circledR}$ ). J Liposome Res 2017, 27 (3), 173-179.

5. López, L.; Vélez, I.; Asela, C.; Cruz, C.; Alves, F.; Robledo, S.; Arana, B., A phase II study to evaluate the safety and efficacy of topical 3\% amphotericin B cream (Anfoleish) for the treatment of uncomplicated cutaneous leishmaniasis in Colombia. PLOS Negl Trop Dis 2018, 12 (7), e0006653.

6. Ganeshpurkar, A.; Vaishya, P.; Jain, S.; Pandey, V.; Bansal, D.; Dubey, N., Delivery of Amphotericin B for Effective Treatment of Candida Albicans Induced Dermal Mycosis in Rats via Emulgel System: Formulation and Evaluation. Indian J Dermatol 2014, 59 (4), 369-374.

7. Serrano, D. R.; Gallagher, K. H.; Healy, A. M., Emerging Nanonisation Technologies: Tailoring Crystalline Versus Amorphous Nanomaterials. Curr Top Med Chem 2015, 15 (22), 2327-2340.

8. $\quad$ Fernández-García, R.; Lalatsa, A.; Statts, L.; Bolás-Fernández, F.; Ballesteros, M. P.; Serrano, D. R., Transferosomes as nanocarriers for drugs across the skin: Quality by design from lab to industrial scale. Int J Pharm 2020, 573, 118817.

9. Cevc, G.; Blume, G., Lipid vesicles penetrate into intact skin owing to the transdermal osmotic gradients and hydration force. Biochim Biophys Acta 1992, 1104 (1), 226-232.

10. Rajan, R.; Jose, S.; Mukund, V. P.; Vasudevan, D. T., Transferosomes - A vesicular transdermal delivery system for enhanced drug permeation. J Adv Pharm Technol Res 2011, 2 (3), 138-143.

11. Singodia, D.; Gupta, G.; Verma, A.; Singh, V.; Shukla, P.; Misra, P.; Sundar, S.; Dube, A.; Mishra, P., Development and Performance Evaluation of Amphotericin B Transfersomes Against Resistant and Sensitive Clinical Isolates of Visceral Leishmaniasis. J Biomed Nanotech 2010, 6, 293302.

12. Ruiz, H. K.; Serrano, D. R.; Dea-Ayuela, M. A.; Bilbao-Ramos, P. E.; Bolas-Fernandez, F.; Torrado, J. J.; Molero, G., New amphotericin B-gamma cyclodextrin formulation for topical use with synergistic activity against diverse fungal species and Leishmania spp. Int J Pharm 2014, 473 (1-2), 148-157.

13. Silveira, F. T.; Lainson, R.; De Castro Gomes, C. M.; Laurenti, M. D.; Corbett, C. E. P., Immunopathogenic competences of Leishmania (V.) braziliensis and L. (L.) amazonensis in American cutaneous leishmaniasis. Parasite Immunol 2009, 31 (8), 423-431.

14. Lalatsa, A.; Patel, P. V.; Sun, Y.; Kiun, C. C.; Karimi, F.; Zekonyte, J.; Emeriewen, K.; Saleh, G. M., Transcutaneous Anaesthetic Nano-enabled Hydrogels for Eyelid Surgery. Int J Pharm 2020, 119003.

15. Kietzmann, M.; Lubach, D.; Heeren, H.J., The mouse epidermis as a model in skin pharmacology: influence of age and sex on epidermal metabolic reactions and their circadian rhythms. Lab Animals 1990, 24 (4), 321-327.

16. Garnier, T.; Mäntylä, A.; Järvinen, T.; Lawrence, J.; Brown, M.; Croft, S., In vivo studies on the antileishmanial activity of buparvaquone and its prodrugs. J Antimicrob Chemother 2007, 60 (4), 802-810.

17. Reddy, M. B.; Stinchcomb, A. L.; Guy, R. H.; Bunge, A. L., Determining Dermal Absorption Parameters in Vivo From Tape Strip Data. Pharm Res 2002, 19 (3), 292-298.

18. Donnelly, R. F.; Singh, T. R.; Garland, M. J.; Migalska, K.; Majithiya, R.; McCrudden, C. M.; Kole, P. L.; Mahmood, T. M.; McCarthy, H. O.; Woolfson, A. D., Hydrogel-Forming Microneedle Arrays for Enhanced Transdermal Drug Delivery. Adv Func Mater 2012, 22 (23), 4879-4890.

19. Kalluri, H.; Kolli, C. S.; Banga, A. K., Characterization of microchannels created by metal microneedles: formation and closure. AAPS J 2011, 13 (3), 473-481.

20. Kim, Y. C.; Park, J. H.; Prausnitz, M. R., Microneedles for drug and vaccine delivery. Adv Drug Deliv Rev 2012, 64 (14), 1547-1568.

21. Esfandiarpour, I.; Farajzadeh, S.; Rahnama, Z.; Fathabadi, E. A.; Heshmatkhah, A., Adverse effects of intralesional meglumine antimoniate and its influence on clinical laboratory parameters in the treatment of cutaneous leishmaniasis. Int J Dermatol 2012, 51 (10), 1221-1225. 
22. Ben Salah, A.; Ben Messaoud, N.; Guedri, E.; Zaatour, A.; Ben Alaya, N.; Bettaieb, J.; Gharbi, A.; Belhadj Hamida, N.; Boukthir, A.; Chlif, S.; Abdelhamid, K.; El Ahmadi, Z.; Louzir, H.; Mokni, M.; Morizot, G.; Buffet, P.; Smith, P. L.; Kopydlowski, K. M.; Kreishman-Deitrick, M.; Smith, K. S.; Nielsen, C. J.; Ullman, D. R.; Norwood, J. A.; Thorne, G. D.; McCarthy, W. F.; Adams, R. C.; Rice, R. M.; Tang, D.; Berman, J.; Ransom, J.; Magill, A. J.; Grogl, M., Topical Paromomycin with or without Gentamicin for Cutaneous Leishmaniasis. N Engl J Med 2013, 368 (6), 524-532.

23. Sosa, N.; Capitán, Z.; Nieto, J.; Nieto, M.; Calzada, J.; Paz, H.; Spadafora, C.; KreishmanDeitrick, M.; Kopydlowski, K.; Ullman, D.; McCarthy, W. F.; Ransom, J.; Berman, J.; Scott, C.; Grogl, M., Randomized, double-blinded, phase 2 trial of WR 279,396 (paromomycin and gentamicin) for cutaneous leishmaniasis in Panama. Am J Trop Med Hyg 2013, 89 (3), 557-563.

24. Kagan, L.; Gershkovich, P.; Wasan, K. M.; Mager, D. E., Physiologically based pharmacokinetic model of amphotericin B disposition in rats following administration of deoxycholate formulation (Fungizone ${ }^{\circledR}$ ): pooled analysis of published data. AAPS J 2011, 13 (2), 255264.

25. Jiang, T.; Wang, T.; Li, T.; Ma, Y.; Shen, S.; He, B.; Mo, R., Enhanced Transdermal Drug Delivery by Transfersome-Embedded Oligopeptide Hydrogel for Topical Chemotherapy of Melanoma. ACS Nano 2018, 12 (10), 9693-9701.

26. Jangdey, M. S.; Gupta, A.; Saraf, S.; Saraf, S., Development and optimization of apigeninloaded transfersomal system for skin cancer delivery: in vitro evaluation. Artif Cells Nanomed Biotechnol 2017, 45 (7), 1452-1462.

27. Morsi, N. M.; Aboelwafa, A. A.; Dawoud, M. H. S., Enhancement of the bioavailability of an antihypertensive drug by transdermal protransfersomal system: formulation and in vivo study. $J$ Liposome Res 2017, 1-12.

28. AEMPS AmBisome, data sheet. https://cima.aemps.es/cima/dochtml/ft/61117/FT 61117.html (accessed 29th December 2019).

29. Kaur, L.; Singh, K.; Paul, S.; Singh, S.; Singh, S.; Jain, S. K., A Mechanistic Study to Determine the Structural Similarities Between Artificial Membrane Strat- $\mathrm{M}^{\mathrm{TM}}$ and Biological Membranes and Its Application to Carry Out Skin Permeation Study of Amphotericin B Nanoformulations. AAPS PharmSciTech 2018, 19 (4), 1606-1624.

30. Joshi, V.; Brewster, D.; Colonero, P., In vitro diffusion studies in transdermal research: A synthetic membrane model in place of human skin. Drug Develop Deliv 2012, 12, 40-42.

31. Uchida, T.; Kadhum, W. R.; Kanai, S.; Todo, H.; Oshizaka, T.; Sugibayashi, K., Prediction of skin permeation by chemical compounds using the artificial membrane, Strat- $\mathrm{M}^{\mathrm{TM}}$. Eur J Pharm Sci 2015, 67, 113-118.

32. Taylor, V. M.; Cedeño, D. L.; Muñoz, D. L.; Jones, M. A.; Lash, T. D.; Young, A. M.; Constantino, M. H.; Esposito, N.; Vélez, I. D.; Robledo, S. M., In vitro and in vivo studies of the utility of dimethyl and diethyl carbaporphyrin ketals in treatment of cutaneous leishmaniasis. Antimicrob Agents Chemother 2011, 55 (10), 4755-4764.

33. Ryder, N. S.; Wagner, S.; Leitner, I., In vitro activities of terbinafine against cutaneous isolates of Candida albicans and other pathogenic yeasts. Antimicrob Agents Chemother 1998, 42 (5), 1057-1061.

34. Morais-Teixeira, E.; Gallupo, M.; Rodrigues, L.; Rabello, A., In vitro interaction between paromomycin sulphate and four drugs with leishmanicidal activity against three New World Leishmania species. J Antimicrob Chemother 2014, 69 (1), 150-154.

35. Hartsel, S.; Bolard, J., Amphotericin B: new life for an old drug. Trends Pharmacol Sci 1996, $17(12), 445-449$.

36. Yardley, V.; Croft, S. L., A comparison of the activities of three amphotericin B lipid formulations against experimental visceral and cutaneous leishmaniasis. Int J Antimicrob Ag 2000, 13 (4), 243-248. 
37. Hong, Y.; Shaw, P. J.; Tattam, B. N.; Nath, C. E.; Earl, J. W.; Stephen, K. R.; McLachlan, A. J., Plasma protein distribution and its impact on pharmacokinetics of liposomal amphotericin $B$ in paediatric patients with malignant diseases. Eur J Clin Pharmacol 2007, 63 (2), 165-172.

38. Ordóñez-Gutiérrez, L.; Espada-Fernández, R.; Dea-Ayuela, M. A.; Torrado, J. J.; BolásFernandez, F.; Alunda, J. M., In vitro effect of new formulations of amphotericin B on amastigote and promastigote forms of Leishmania infantum. Int J Antimicrob Ag 2007, 30 (4), 325-329.

39. Dar, M.; McElroy, C.; Khan, M.; Satoskar, A.; Khan, G. M., Development and evaluation of novel miltefosine- polyphenol co-loaded second generation nano- transfersomes for the topical treatment of cutaneous leishmaniasis. Expert Opin Drug Del 2020, 17 (1), 97-110.

40. González, K.; Diaz, R.; Ferreira, A. F.; García, V.; Paz, H.; Calzada, J. E.; Ruíz, M.; Laurenti, M.; Saldaña, A., Histopathological characteristics of cutaneous lesions caused by Leishmania Viannia panamensis in Panama. Rev Inst Med Trop SP 2018, 60:e8.

41. Gupta, J.; Gill, H. S.; Andrews, S. N.; Prausnitz, M. R., Kinetics of skin resealing after insertion of microneedles in human subjects. J Control Rel 2011, 154 (2), 148-155.

42. Kalluri, H.; Kolli, C. S.; Banga, A. K., Characterization of Microchannels Created by Metal Microneedles: Formation and Closure. AAPS J 2011, 13 (3), 473-481.

43. Van Bocxlaer, K.; Yardley, V.; Murdan, S.; Croft, S. L., Drug permeation and barrier damage in Leishmania-infected mouse skin. J Antimicrob Chemother 2016, 71 (6), 1578-85.

44. Ekanayake-Mudiyanselage, S.; Aschauer, H.; Schmook, F. P.; Jensen, J. M.; Meingassner, J. G.; Proksch, E., Expression of epidermal keratins and the cornified envelope protein involucrin is influenced by permeability barrier disruption. J Invest Dermatol 1998, 111 (3), 517-23.

45. ShinEtsu Metolose, data sheet. http://www.metolose.jp/en/pharmaceutical/metolose.html (accessed 14th January 2020).

46. Rodrigues, C.; Khalil, N.; Mainardes, R., Determination of amphotericin B in PLA-PEG blend nanoparticles by HPLC-PDA. Braz J Pharm Sci 2014, 50, 859-868.

47. Serrano, D. R.; Walsh, D.; O'Connell, P.; Mugheirbi, N. A.; Worku, Z. A.; Bolas-Fernandez, F.; Galiana, C.; Dea-Ayuela, M. A.; Healy, A. M., Optimising the in vitro and in vivo performance of oral cocrystal formulations via spray coating. Eur J Pharm Biopharm 2018, 124, 13-27.

48. Espada, R.; Josa, J. M.; Valdespina, S.; Dea, M. A.; Ballesteros, M. P.; Alunda, J. M.; Torrado, J. J., HPLC assay for determination of amphotericin B in biological samples. BMC 2008, 22 (4), 402-407.

49. Millipore, M. Strat-M membrane. https://www.incosmetics.com/ novadocuments/255210?v=636053819429970000 (accessed July 18, 2019). 50. Serrano, D. R.; Gordo, M. J.; Matji, A.; Gonzalez, S.; Lalatsa, A.; Torrado, J. J., Tuning the Transdermal Delivery of Hydroquinone upon Formulation with Novel Permeation Enhancers. Pharmaceutics 2019, 11 (4), 167.

51. Lalatsa, A.; Emeriewen, K.; Protopsalti, V.; Skelton, G.; Saleh, G. M., Developing transcutaneous nanoenabled anaesthetics for eyelid surgery. Brit J Ophthalmol 2016, 100 (6), 871 876.

52. Kaur, L.; Singh, K.; Paul, S.; Singh, S.; Singh, S.; Jain, S. K., A Mechanistic Study to Determine the Structural Similarities Between Artificial Membrane Strat-M and Biological Membranes and Its Application to Carry Out Skin Permeation Study of Amphotericin B Nanoformulations. AAPS PharmSciTech 2018. 19 (4), 1606-1624.

53. Lalatsa, A.; Statts, L.; de Jesus, J.A.; Adewusi, O.; Dea-Ayuela, M.A.; Bolas-Fernandez, F.; Dalastra, M.; Domingues, L.F.; Serrano, D.R. Topical Buparvaquone Nano-enabled Hydrogels for Cutaneous Leishmaniasis Int J Pharm 2020, 119734.

54. Evans, B. C.; Nelson, C. E.; Yu, S. S.; Beavers, K. R.; Kim, A. J.; Li, H.; Nelson, H. M.; Giorgio, T. D.; Duvall, C. L., Ex vivo red blood cell hemolysis assay for the evaluation of $\mathrm{pH}$-responsive endosomolytic agents for cytosolic delivery of biomacromolecular drugs. J Vis Exp 2013, (73), e50166. 
55. Dea-Ayuela, M. A.; Castillo, E.; Gonzalez-Alvarez, M.; Vega, C.; Rolon, M.; BolasFernandez, F.; Borras, J.; Gonzalez-Rosende, M. E., In vivo and in vitro anti-leishmanial activities of 4nitro-N-pyrimidin- and N-pyrazin-2-ylbenzenesulfonamides, and N2-(4-nitrophenyl)-N1propylglycinamide. Bioorg Med Chem 2009, 17 (21), 7449-7456.

56. Bilbao-Ramos, P.; Sifontes-Rodriguez, S.; Dea-Ayuela, M. A.; Bolas-Fernandez, F., A fluorometric method for evaluation of pharmacological activity against intracellular Leishmania amastigotes. J Microbiol Methods 2012, 89 (1), 8-11.

\section{For Table of Contents Use Only}

\section{Ultra-deformable lipid vesicles localise Amphotericin B in the dermis for the treatment of skin infectious diseases}

Raquel Fernández-García ${ }^{1 \#}$, Larry Statts ${ }^{2 \#}$, Jéssica A. de Jesus ${ }^{3}$, Maria Auxiliadora Dea-Ayuela ${ }^{4}$, Liliana Bautista ${ }^{2}$, Rúben Simão ${ }^{1}$, Francisco Bolás-Fernández ${ }^{5}$, Maria Paloma Ballesteros ${ }^{1,6}$, Marcia Dalastra Laurenti ${ }^{3}$, Luiz F. D. Passero ${ }^{7,8}$, Aikaterini Lalatsa ${ }^{2 *}$, Dolores R. Serrano ${ }^{1,6 *}$. 
Amphotericin B encapsulated within transfersomes enhances in vitro skin permeation resulting in therapeutic levels after in vivo administration and a significant reduction in Leishmania parasites in mice infected with cutaneous leishmaniasis. This formulation is readily translatable as a good therapeutic alternative to current treatments for cutaneous leishmaniasis and fungal infections in developed and developing countries.

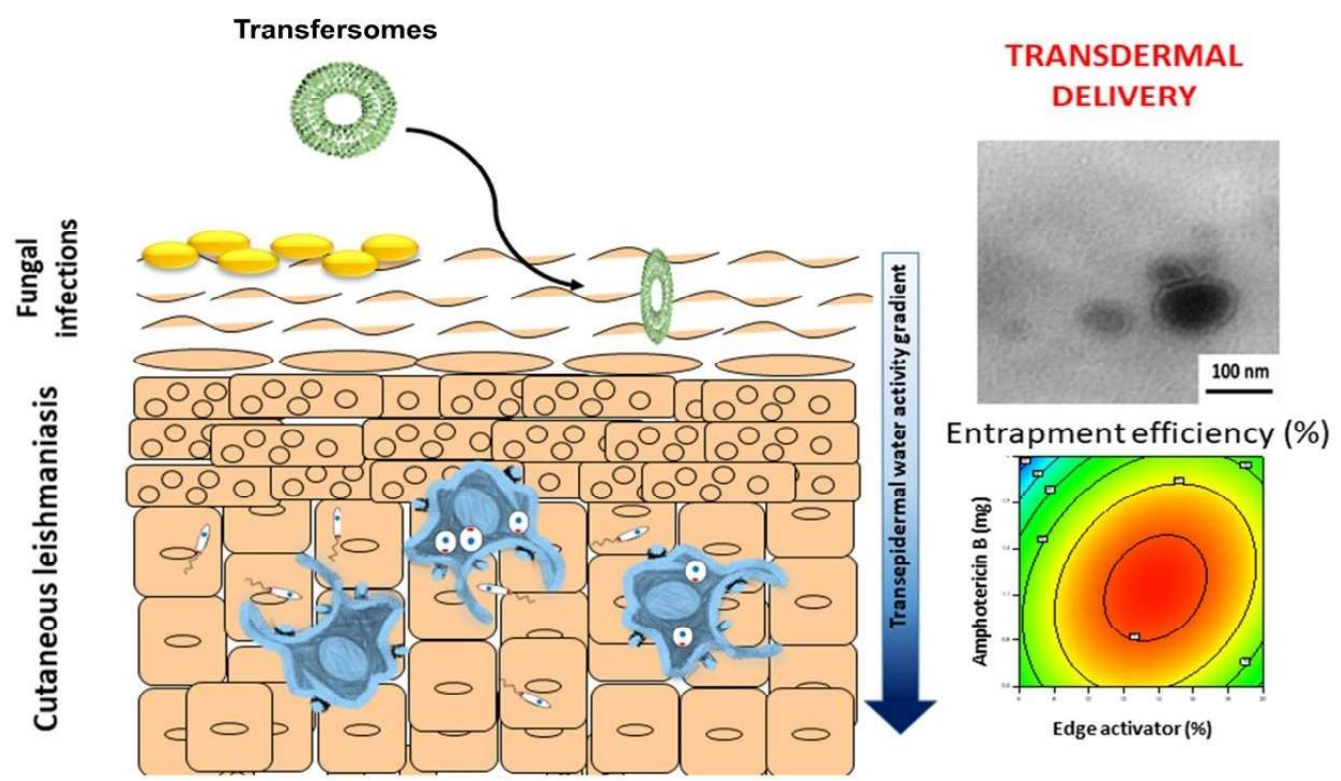



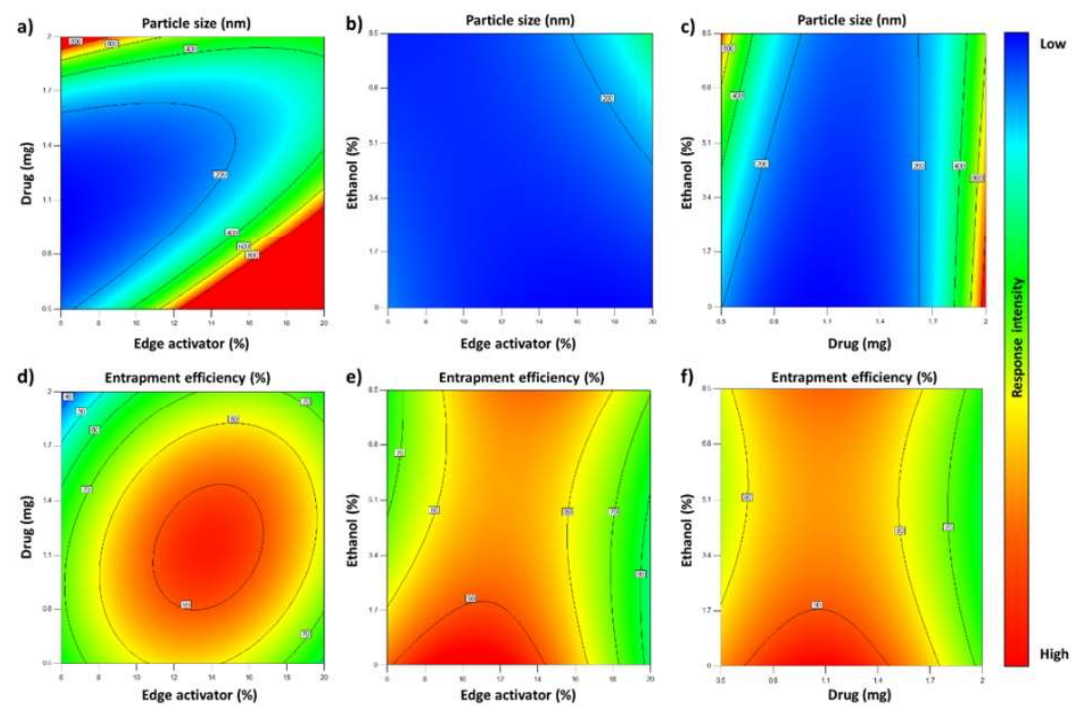

Figure 1: 2D Contour plots showing the effect of the amount of edge activator, drug and ethanol on particle size $(a, b, c)$ and EE $(d, e, f)$.

$338 \times 190 \mathrm{~mm}(96 \times 96 \mathrm{DPI})$ 

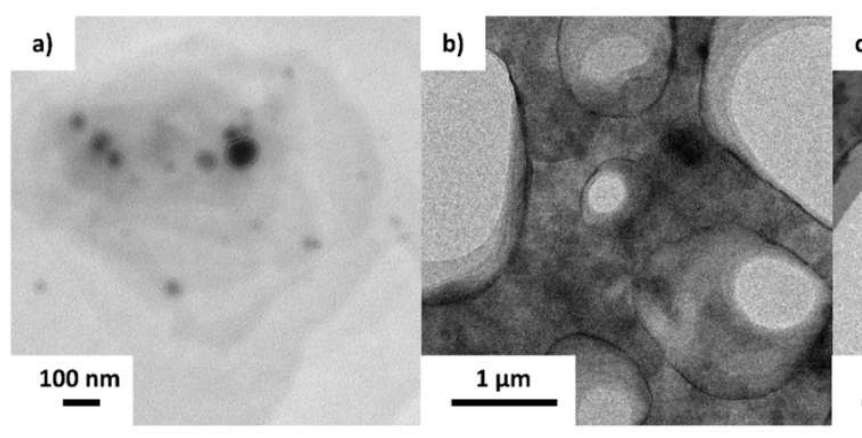

c)

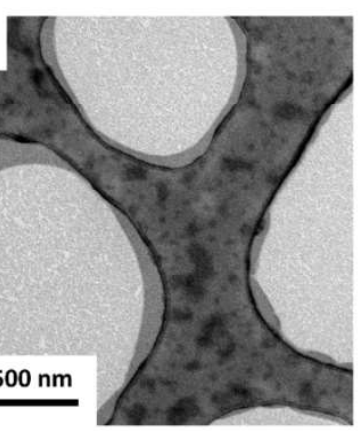

Figure 2: TEM micrographs of AmB-TFs; a) Freshly prepared AmB-TFs stained with $1 \%$ uranyl acetate (Bar: $100 \mathrm{~nm})$; b) Reconstituted freshly prepared freeze-dried AmB-TFs in deionised water stained with $2 \%$ phosphotungstic acid (Bar: $1 \mu \mathrm{m}$ ); c) AmB-TFs after 28 days of storage at $4{ }^{\circ} \mathrm{C}$ stained with $2 \%$ phosphotungstic acid (Bar: $500 \mathrm{~nm}$ ).

$338 \times 190 \mathrm{~mm}(96 \times 96 \mathrm{DPI})$ 

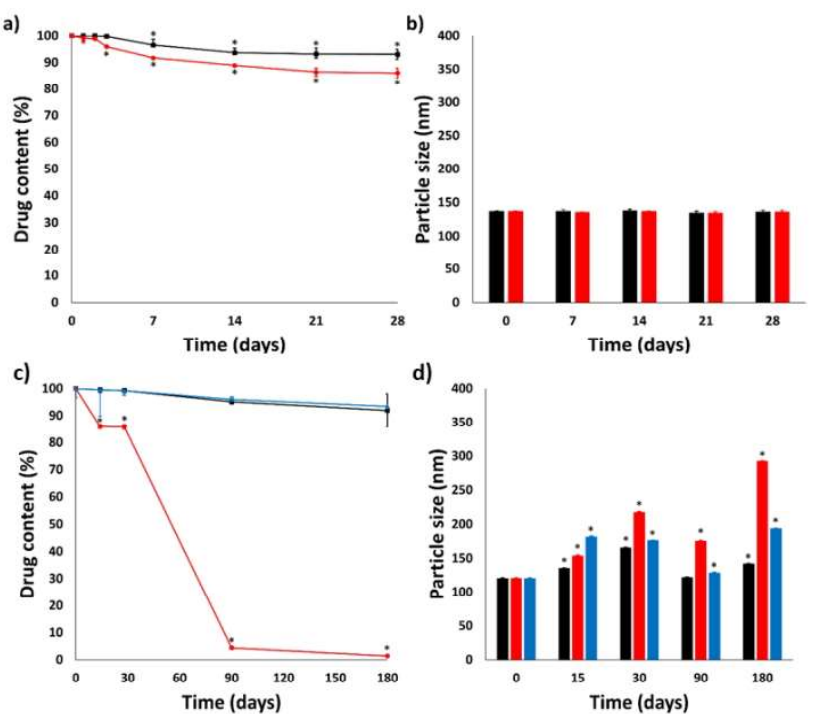

d) ${ }_{400}$

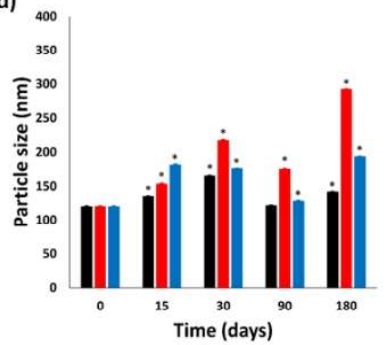

Figure 3: Physicochemical stability of liquid and freeze-dried AmB-TFs: a) Chemical stability of liquid AmBTFs; b) Physical stability of liquid AmB-TFs. Key: $25{ }^{\circ} \mathrm{C}$ (red circles), $4{ }^{\circ} \mathrm{C}$ (black squares); $* p<0.05$ is indicative of statistically significant difference between each time point and time 0 (one-way ANOVA posthoc Dunnett's test). c) Chemical stability of freeze-dried AmB-TFs; d) Physical stability of freeze-dried AmBTFs. Key: $25{ }^{\circ} \mathrm{C}, 10 \% \mathrm{RH}$ (black squares), $25{ }^{\circ} \mathrm{C}, 60 \% \mathrm{RH}$ (red circles), $4{ }^{\circ} \mathrm{C}, 10 \% \mathrm{RH}$ (blue triangles); $*_{p}<0.05$ is indicative of statistically significant difference between each time point and time 0 (one-way ANOVA post-hoc Dunnett's test).

$338 \times 190 \mathrm{~mm}(96 \times 96 \mathrm{DPI})$ 


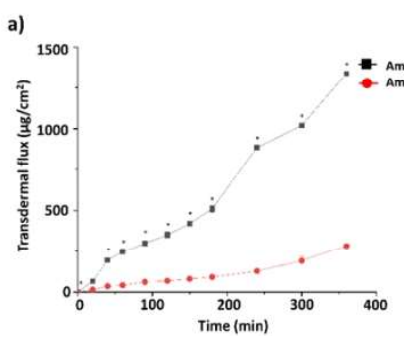

b)

c)

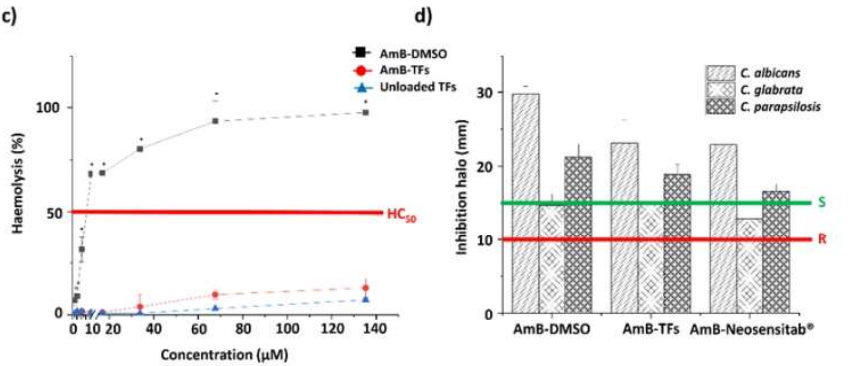

Figure 4: Permeability, toxicity and in vitro activity of AmB-TFs: a) Permeability of AmB-TFs and AmB-DMSO across Strat-M® membranes; b) Permeability of AmB-TFs and AmB-DMSO across mouse skin; c) Haemolytic toxicity of AmB-TFs, AmB-DMSO and unloaded TFs; d) Antifungal activity of AmB-TFs, AmB-DMSO and AmB-Neosensitabs $\AA$. Key: AmB-DMSO (black squares), AmB-TFs (red circles), unloaded TFs (blue triangles), concentration that produces $50 \%$ of haemolysis (HC50), sensitivity (S), resistance (R). Statistically significant difference is represented by $*(p<0.05)$ when comparing to AmB-DMSO with other formulations

$338 \times 190 \mathrm{~mm}(96 \times 96$ DPI) 

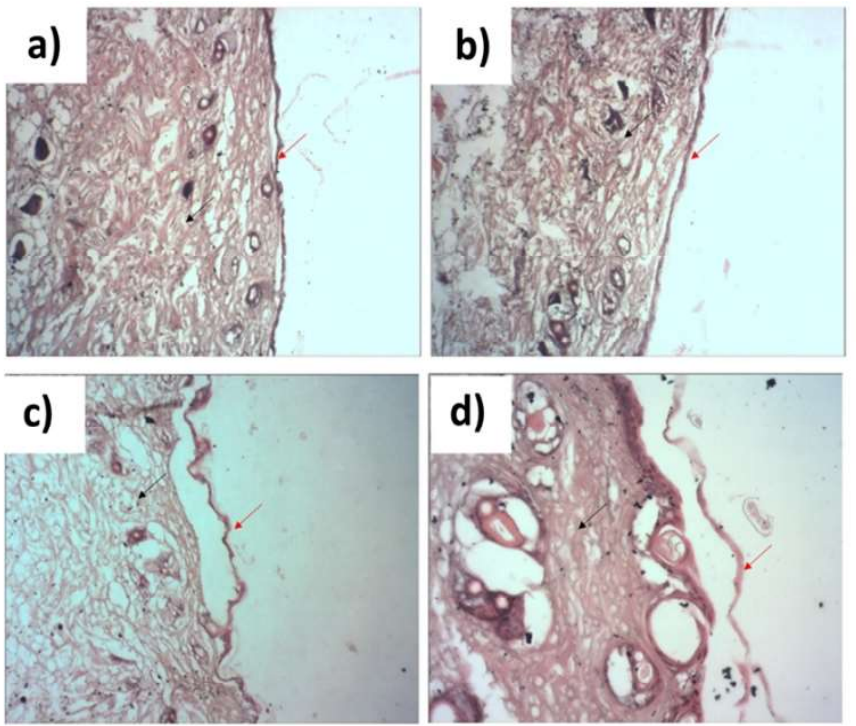

Figure 5: Micrographs of histological studies for treated skin with AmB -TFs and AmB dissolved in DMSO: a) mouse skin after exposure to AmB-TFs, 4x magnification; b) mouse skin after exposure to AmB-TFs, 10x magnification; c) mouse skin after exposure to AmB-DMSO, 4x magnification; d) mouse skin after exposure to AmB-DMSO; 10x magnification.

$338 \times 190 \mathrm{~mm}(96 \times 96 \mathrm{DPI})$ 
a)

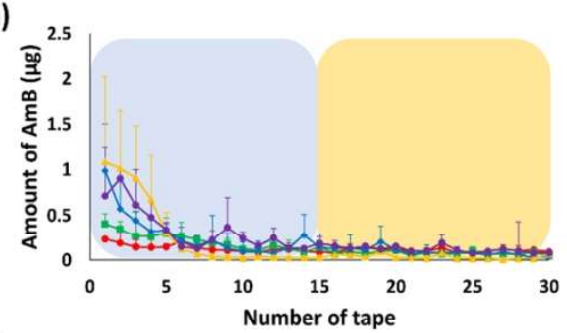

c)

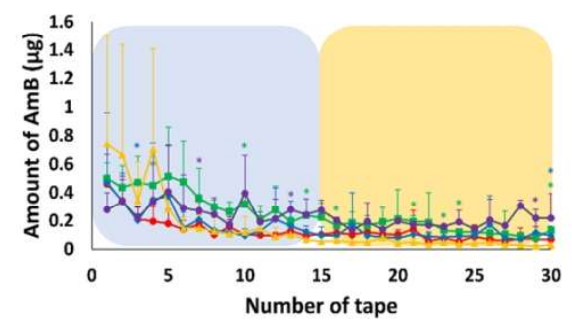

b)

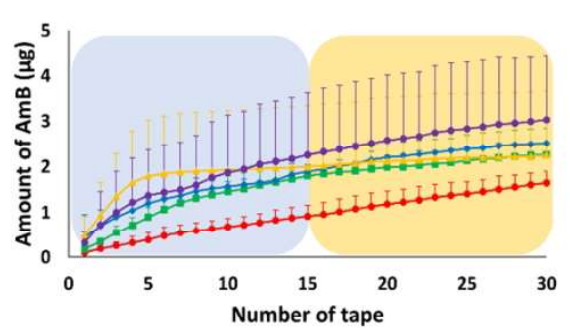

d)

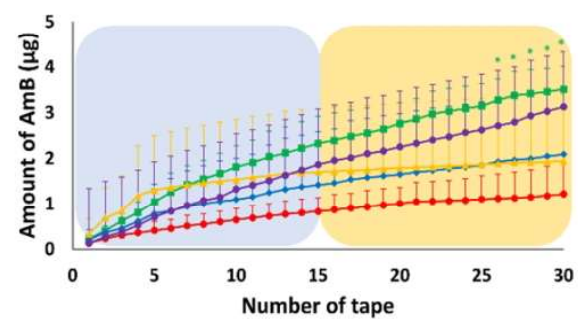

Figure 6: Averaged amount of AmB per tape strip (a,c) and cumulative amount of AmB (b, d) in mouse skin after $0.5,1,2,4$ and $6 \mathrm{~h}$ of post-administration without the application of microneedles $(\mathrm{a}, \mathrm{b})$ and after the utilization of microneedles (c, d). Key: $0.5 \mathrm{~h}$ (red circles), $1 \mathrm{~h}$ (green squares), $2 \mathrm{~h}$ (blue diamonds), $4 \mathrm{~h}$ (yellow triangles), $6 \mathrm{~h}$ (purple circles). The blue and orange panels represent the stratum corneum and the evaluated skin once the stratum corneum is removed, respectively. Statistically significant differences are represented by * $(p<0.05$, one way-ANOVA post-hoc Tukey's test).

$338 \times 190 \mathrm{~mm}(96 \times 96 \mathrm{DPI})$ 
a)
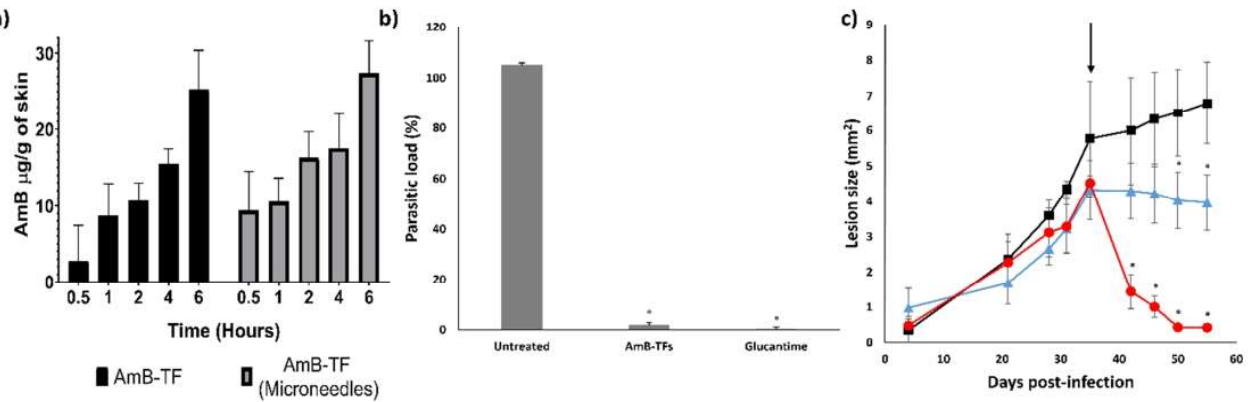

Figure 7. a) Amount of remaining AmB in the skin after removal of 30 tape-strips after the topical AmB-TFs administration (black bars) and topical AmB-TFs administration after application of microneedles (grey bars); b) In vivo antiparasitic activity (parasitic load) of AmB-TFs $(0.5 \mathrm{mg} / \mathrm{ml}, 20 \mathrm{mg}$ of formulation/day, once daily topically applied) and Glucantime $\AA(50 \mathrm{mg} / \mathrm{kg}$, once daily $25 \mu$ intralesionally administered) in experimental cutaneous leishmaniasis caused by L. (L.) amazonensis; c) Evaluation of lesion size during the

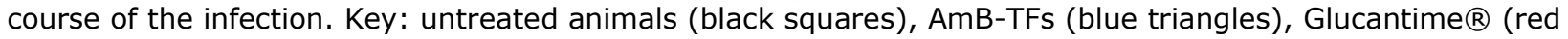
circles). Arrow indicates the onset of treatment. Statistically significant differences between treated and untreated animals is represented by $*(p<0.05$, Mann-Whitney $U$ test).

$338 \times 190 \mathrm{~mm}(300 \times 300 \mathrm{DPI})$ 